\title{
APOE Genotype Modifies the Plasma Oxylipin Response to Omega-3 Polyunsaturated Fatty Acid Supplementation in Healthy Individuals
}

\author{
Rasha N. M. Saleh ${ }^{1,2 \star}$, Annette L. West ${ }^{3}$, Annika I. Ostermann ${ }^{4}$, Nils Helge Schebb ${ }^{4}$, \\ Philip C. Calder ${ }^{3,5}$ and Anne Marie Minihane ${ }^{1}$
}

\begin{abstract}
${ }^{1}$ Nutrition and Preventive Medicine Group, Norwich Medical School, University of East Anglia, Norwich, United Kingdom, ${ }^{2}$ Department of Clinical and Chemical Pathology, Faculty of Medicine, Alexandria University, Alexandria, Egypt, ${ }^{3}$ School of Human Development and Health, Faculty of Medicine, University of Southampton, Southampton, United Kingdom, ${ }^{4}$ Chair of Food Chemistry, Faculty of Mathematics and Natural Sciences, University of Wuppertal, Wuppertal, Germany, ${ }^{5}$ National Institute for Health Research (NIHR) Southampton Biomedical Research Centre, University Hospital Southampton NHS Foundation Trust and University of Southampton, Southampton, United Kingdom
\end{abstract}

\section{OPEN ACCESS}

Edited by: Fredrik Rosqvist,

Uppsala University, Sweden

Reviewed by:

Carla Taylor,

University of Manitoba, Canada Rosalind Fallaize

University of Hertfordshire, United Kingdom

*Correspondence: Rasha N. M. Saleh r.saleh@uea.ac.uk

Specialty section: This article was submitted to

Nutrition and Metabolism,

a section of the journal

Frontiers in Nutrition

Received: 11 June 2021

Accepted: 17 August 2021

Published: 17 September 2021

Citation:

Saleh RNM, West AL, Ostermann Al,

Schebb NH, Calder PC and Minihane AM (2021) APOE Genotype Modifies the Plasma Oxylipin Response to Omega-3

Polyunsaturated Fatty Acid

Supplementation in Healthy Individuals. Front. Nutr. 8:723813.

doi: 10.3389/fnut.2021.723813
The omega-3 polyunsaturated fatty acids ( $\mathrm{n}-3$ PUFAs), eicosapentaenoic acid (EPA) and docosahexaenoic acid (DHA), mediate inflammation in large part by affecting pro-inflammatory and anti-inflammatory/pro-resolving oxylipin concentrations. Common gene variants are thought to underlie the large inter-individual variation in oxylipin levels in response to n-3 PUFA supplementation, which in turn is likely to contribute to the overall heterogeneity in response to n-3 PUFA intervention. Given its known role in inflammation and as a modulator of the physiological response to EPA and DHA, here we explore, for the first time, the differential response of plasma hydroxy-, epoxy- and dihydroxy-arachidonic acid, EPA and DHA oxylipins according to apolipoprotein E (APOE) genotype using samples from a dose-response parallel design RCT. Healthy participants were given doses of EPA+DHA equivalent to intakes of 1, 2, and 4 portions of oily fish per week for 12 months. There was no difference in the plasma levels of EPA, DHA or ARA between the wildtype APOE3/E3 and APOE4 carrier groups after 3 or 12 months of n-3 PUFA supplementation. At 12 months, hydroxy EPAs (HEPEs) and hydroxy-DHAs (HDHAs) were higher in APOE4 carriers, with the difference most evident at the highest EPA+DHA intake. A significant APOE*n-3 PUFA dose effect was observed for the CYP- $\omega$ hydroxylase products 19-HEPE $(p=0.027)$ and 20-HEPE $(p=0.011)$. 8-HEPE, which, along with several other plasma oxylipins, is an activator of peroxisome proliferator activated receptors (PPARs), showed the highest fold change in APOE4 carriers (14-fold) compared to APOE3/E3 (4-fold) $(p=0.014)$. Low basal plasma EPA levels (EPA $<0.85 \%$ of total fatty acids) were associated with a greater change in 5-HEPE, 9-HEPE, 11-HEPE, and 20-HEPE compared to high basal EPA levels (EPA $>1.22 \%$ of total fatty acids). In conclusion, $A P O E$ genotype modulated the plasma oxylipin response to increased EPA+DHA intake, with APOE4 carriers presenting with the greatest increases following high dose n-3 PUFA supplementation for 12 months.

Keywords: APOE, oxylipins, PUFAs, EPA, DHA, HDHA, HEPE, PPAR 


\section{INTRODUCTION}

The omega-3 polyunsaturated fatty acids ( $\mathrm{n}-3$ PUFAs) eicosapentaenoic acid (EPA) and docosahexaenoic acid (DHA) have long been known to play a role in promoting human health and well-being (1). Higher EPA and DHA intake is associated with a lower risk of cardiovascular disease and mortality (2,3), cognitive decline (4), rheumatoid arthritis (5), obesity (6), and overall mortality (7). The biological actions of n-3 PUFAs are partly mediated through their oxidized metabolites, called oxylipins. Oxylipins are formed via three main pathways involving cyclooxygenases, lipoxygenases, and several cytochrome P450 (CYP) enzymes which produce hydroxy-, dihydroxy-, or epoxy- fatty acids (FAs) among other products. Due to their highly unsaturated status, PUFAs can also be non-enzymatically oxidized (i.e., autooxidation) by reactive oxygen and nitrogen species, to produce a number of oxylipins (Figure 1) $(8,9)$. Oxylipins are potent lipid mediators of multiple physiological processes (10). Epoxy-arachidonic acid (ARA) species (EpETrEs), products of CYP2C and 2J epoxygenases, have recently been shown to have cardiovascular $(11,12)$ and anti-inflammatory benefits (13). Epoxy-EPAs (EpETEs) and -DHAs (EpDPEs) are anti-arrhythmic (14) and inhibit angiogenesis (15). Epoxy-FAs are metabolized by hydration to the corresponding less active dihydroxy-FAs by the action of soluble epoxide hydrolase ( $\mathrm{sEH}$ ) (9). As a result, the ratio of dihydroxy- to epoxy-FAs has been used as an indicator of sEH activity (16). Hydroxy-ARAs (HETEs), -EPAs (HEPEs), and -DHAs (HDHAs) have a wide range of functions, for example regulating neutrophil chemotaxis, platelet aggregation and adipogenesis (9). 8-HEPE has recently been found to reduce plasma LDL-cholesterol and triglycerides in obese mice through binding to peroxisome proliferation activator receptors (PPARs) (17). Other hydroxy-FAs, such as 18-HEPE and 17-HDHA are precursors for specialized pro-resolving mediators (SPMs). SPMs (including resolvins, protectins, maresins, and lipoxins) are now known for their anti-inflammatory and pro-resolving roles (18).

Several intervention studies have shown a rise in EPA- and DHA-derived and a fall in ARA-derived oxylipins in response to n-3 PUFA supplementation (19-23). This response is linearly related to n-3 PUFA dose (24). However, despite the high compliance to n-3 PUFA treatment in most studies, a strong inter-individual variation in the oxylipin response to different doses of n-3 PUFA intervention was observed $(23,25,26)$. This variation has been partly explained by differences in baseline EPA and DHA status. Individuals with lower basal levels of EPA and DHA levels showed a greater increase in the n-3 PUFA-derived oxylipins in response to increased n-3 PUFA intake $(23,25)$. Genetic variation in enzymes involved in PUFA metabolism have been implicated as another possible cause of variation in the oxylipin response to n-3 PUFAs. Genetic variation in $\mathrm{LTA}_{4} \mathrm{H}$, an enzyme in the pathway of leukotriene synthesis, significantly interacted with dietary intake of n-3 and n- 6 fatty acids to determine intima-media thickness (IMT) in one population (27). In another study, variants in ALOX5 gene were associated with a differential oxylipin response to fish oil supplementation (28).
Apolipoprotein E (APOE) regulates the concentrations and metabolism of cholesterol and PUFAs in the circulation and in tissues (29). The APOE gene has three allele variants $\varepsilon 2, \varepsilon 3$, and $\varepsilon 4$, determined by two SNPs, rs429358 at codon 112 and rs7412 at codon 158. The frequency of the major APOE3 allele ranges from 48 to $94 \%$ while the APOE4 allele has a wider global range (3-41\%) (30). APOE genotype has long been known to affect the response to n-3 PUFA interventions in healthy participants (31) and in patients with cardiovascular (32) and cognitive disorders (33). Studies have investigated the benefit of APOE4-targeted dietary approaches on blood lipid levels $(34,35)$ and Alzheimer's disease risk $(36,37)$. Despite APOE genotype being a known modulator of response to n-3 PUFA interventions, the mechanistic basis for this is poorly understood, and the effect of APOE genotype on oxylipin responses to increased n-3 PUFA intake has not been investigated. In this study, we hypothesize that the change in the plasma concentrations of free oxylipins in response to n-3 PUFA supplementation will differ according to $A P O E$ genotype. To test this hypothesis, we genotyped healthy subjects who participated in a well-designed randomized control trial (RCT), where EPA and DHA capsules were given in different doses to mimic three different patterns of oily fish intake for a duration of 12 months. Plasma phosphatidylcholine (PC) fatty acids and free oxylipin concentrations were measured at baseline, 3 and 12 months, as reported elsewhere $(24,38)$.

\section{METHODS}

The primary aim of the RCT was to investigate the time course and dose-response effect of EPA and DHA supplementation on the EPA and DHA content of different blood and tissue pools (38); a secondary a posteriori aim was to determine the effect on plasma oxylipin concentrations (24). Here we investigate if $A P O E$ genotype influences habitual plasma oxylipin concentrations and their response to EPA and DHA supplementation. The current analysis is considered as exploratory.

\section{Participants and Study Design}

The study was a double-blinded, parallel RCT in healthy subjects with low habitual fish intake. The study protocol and all procedures and analyses were approved by the Suffolk Local Research Ethics Committee (approval 05/Q0102/181) with the participant consent process allowing for additional analysis of the data collected or biobanked samples. The trial is registered at www.controlled-trials.com as ISRCTN48398526. The study design and the characteristics of the study participants have been described elsewhere (38). Briefly, 163 participants were given EPA+DHA (as triglycerides) in capsules with weekly doses equivalent to the consumption of $0,1,2$, or 4 portions of fatty fish per week, with one portion being equivalent to $3.27 \mathrm{~g}$ EPA + DHA (1:1.2, wt:wt). The period of supplementation was 12 months and blood was sampled at baseline, 3 months and 12 months. Buffy coat and plasma were prepared. For the current analysis, a subset of 110 subjects with APOE genotype data were selected according to the availability of a buffy coat for DNA extraction and $A P O E$ genotyping. The characteristics of the study 


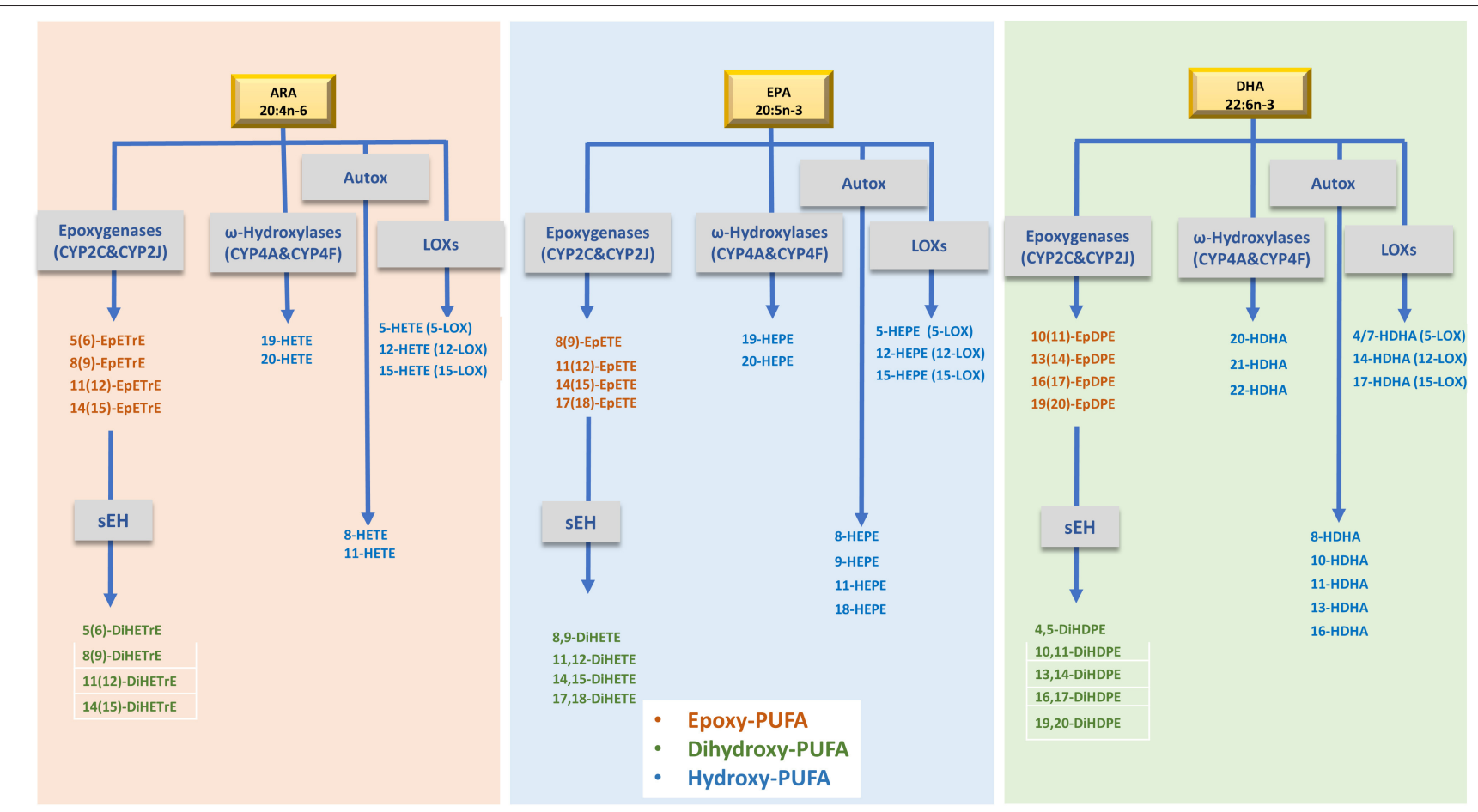

FIGURE 1 | Simplified metabolism of ARA, EPA and DHA by, LOXs and CYPs to produce the oxylipins evaluated in this study. ARA, Arachidonic acid; EPA, Eicosapentaenoic acid; DHA, docosahexaenoic acid; LOXs, lipoxygenases; sEH, soluble epoxide hydrolase enzyme; EpETrE, epoxyeicosatrienoic acid; DiHETrE, dihydroxyeicosatrienoic acid; HETE, hydroxy-eicosatetraenoic acid; EpETE, epoxyeicosatetraenoic acid; DiHETE, dihydroxyeicosatetraenoic acids; HEPE, hydroxyeicosapentaenoic acid; EpDPE, epoxydocosapentaenoic acid; DiHDPE, dihydroxydocosapentaenoic acid; HDHA, hydroxydocosahexaenoic acid. Of note, several oxylipins can be formed by different routes as well as by chemical autoxidation.

TABLE 1 | Basic characteristics of the study population at baseline based on APOE genotype.

\begin{tabular}{|c|c|c|c|}
\hline & APOE2 $(n=18)$ & APOE3 $(n=66)$ & APOE4 $(n=26)$ \\
\hline Age (years) & $55.2 \pm 15.1^{a}$ & $49.6 \pm 15.9^{a}$ & $48.5 \pm 13.8^{a}$ \\
\hline Gender (M/F) & $6 / 12$ & $35 / 31$ & $12 / 14$ \\
\hline BMI $\left(\mathrm{kg} / \mathrm{m}^{2}\right)$ & $24.9 \pm 3.7^{a}$ & $25.7 \pm 3.8^{a}$ & $25.3 \pm 4.3^{a}$ \\
\hline
\end{tabular}

${ }^{a}$ Mean $\pm S D$.

population based on their $A P O E$ genotype are presented in Table 1.

\section{DNA Extraction and APOE Genotyping}

DNA was extracted from the buffy coat of whole blood using the QiAmp DNA Blood Mini kit (Qiagen, UK). The quality and quantity of extracted DNA was checked using a Nanodrop 2000 spectrophotometer. Samples with a yield of at least $15 \mathrm{ng} / \mu \mathrm{l}$ and a $260 / 280$ ratio of at least 1.8 were used for subsequent genotyping.

APOE genotyping was performed by LGC Genomics Ltd, Hoddesdon, UK, using the KASP technology. Primers were designed for the two single-nucleotide polymorphisms (SNPs) in the APOE gene; rs429358 at codon 112 and rs7412 at codon 158. These two SNPs determine the APOE2, E3, and E4 alleles.

\section{Oxylipin Analysis}

Plasma free oxylipins were measured at baseline, 3 and 12 months of n-3 PUFA supplementation. Oxylipin analysis was performed as described elsewhere $(24,39)$. Briefly, oxylipins were isolated from plasma using Bond Elut Certify II Cartridges (Agilent) and analyzed by liquid chromatography-tandem mass spectrometry (LC-MS/MS) after negative electrospray ionization in scheduled selected reaction monitoring.

Fifty EPA-, DHA-, and ARA-derived oxylipins were included in the present study: 9 hydroxy-EPAs (HEPEs), 4 dihydroxyEPAs (DiHETEs), 3 epoxy-EPAs (EpETEs), 11 hydroxy-DHAs (HDHAs), 5 dihydroxy-DHAs (DiHDPEs), 4 epoxy-DHAs (EpDPEs), 6 hydroxy-ARAs (HETEs), 4 dihydroxy-ARAs (DiHETrEs), and 4 epoxy-ARAs (EpETrEs). The concentrations of all HEPEs, DiHETEs, EpETEs, HDHAs, DiHDPEs, EpDPEs, HETEs, DiHETrEs, and EpETrEs covered by the analytical 
TABLE 2 | APOE genotype and allele frequencies in the study population.

\begin{tabular}{|c|c|c|c|}
\hline APOE genotype & Number & Genotype Frequency (\%) & Allele frequency (\%) \\
\hline E2/E2 & 2 & 1.8 & $E 2=16.4$ \\
\hline E2/E3 & 15 & 13.6 & \\
\hline E2/E4 & 1 & 0.9 & \\
\hline E3/E3 & 66 & 60.0 & $\mathrm{E} 3=60.0$ \\
\hline E3/E4 & 25 & 22.7 & $E 4=23.6$ \\
\hline
\end{tabular}

method were summed from the individual data as described previously (24).

\section{Fatty Acids Analysis}

ARA, EPA, and DHA were measured in the plasma phosphatidylcholine (PC) fraction as described previously (38). Briefly, total lipid was extracted from plasma using chloroform:methanol (2:1) Plasma lipid fractions were separated and isolated by solid-phase extraction on aminopropylsilica cartridges. PC, which is the major plasma phospholipid, was eluted with chloroform:methanol (60:40, vol:vol). Fatty acid methyl esters (FAMEs) were formed by transesterification with methanol in sulphuric acid and were separated using gas chromatography. FAMEs were identified by comparison with authentic standards. Fatty acids are expressed as weight percent of total fatty acids in plasma PC.

\section{Statistics and Data Analysis}

Data for fatty acids, oxylipins, and $A P O E$ genotyping were processed using RStudio. Results are presented as mean \pm SEM. The absolute changes in oxylipin concentration after 12 months of supplementation were calculated as $\operatorname{conc}(\mathrm{t} 12)$-conc(t0). Relative changes after 12 months were calculated as conc(t12)/conc(t0). Percent relative change of EPA and DHA and their derived oxylipins were calculated as conc $(\mathrm{t} 12) / \operatorname{conc}(\mathrm{t} 0)^{*} 100$.

Variables were checked for normality using the Shapiro-Wilk test. For normally distributed variables, an independent sample $t$ test was used to test for significance between APOE3 (E3/E3) and APOE4 (E3/E4 + E4/E4) groups. For not-normally distributed variables, the Mann-Whitney test was used. Being aware of the unequal sample sizes between $A P O E$ groups, Levene's test for homogeneity of variances was conducted. Log transformation was performed when required.

A univariate general linear model was used to investigate the main and combined (interaction) effect of $A P O E$ genotype and n-3 PUFA dose on the absolute change of individual oxylipin concentrations at 12 months. Age, sex, BMI, and baseline parent n-3 PUFA concentration were used as covariates.

To investigate the effect of $A P O E$ genotype on the change in oxylipin concentration over time, a repeated measure analysis of oxylipins was performed using the baseline, 3 and 12 month data. A model was built to identify the independent effect of $A P O E$ genotype and dose as main effects, and "APOE* dose" interaction effect. Age, sex and BMI were used as covariates in the model. Time was used as the "within subject" factor. Due to the exploratory nature of this study, correction for multiple testing was not performed and no formal power calculation was carried out, although a retrospective power calculation indicates that for the sum of EPA-, DHA-, and ARA- oxylipins we had 93.6, 91.2 , and $53.8 \%$ power, respectively, to detect a $5 \%$ difference between genotype groups when on the highest dose of n-3 supplementation (4 portions).

All the statistics were carried out using SPSS version 24 (IBM).

\section{RESULTS}

\section{APOE Genotyping Frequencies in the Studied Population}

rs429358 and rs7412 were genotyped from the DNA of 110 participants. Basic characteristics of the study population at baseline based on APOE genotype are shown in Table 1. Genotype and allele frequencies, as shown in Table 2, correspond to the frequencies in the European population (39). Using PLINK software (https://www.cog-genomics.org/plink2), all three APOE genotypes were found to be in Hardy-Weinberg equilibrium. Due to the small number of samples with the E2 allele, with numbers of $<3$ per n-3 PUFA dose group, E2 was excluded from the analysis. Results for E3 (E3/E3 genotype) and E4 (E3/E4 and $\mathrm{E} 4 / \mathrm{E} 4)$ alleles are thus calculated and displayed hereafter.

\section{Baseline Plasma Levels of ARA, EPA, and DHA, and Their Derived Oxylipins}

At baseline, there was no significant effect of $A P O E$ genotype on plasma PC levels of ARA, EPA, or DHA, or their derived oxylipins except for 11-HDHA and 20-HETE, which were lower in the APOE4 group ( $p=0.035$ and $p=0.04$, respectively) (Table 3).

\section{Changes in Parent PUFAs and Their Derived Plasma Oxylipins Following Supplementation}

There was no difference in the level of plasma PC EPA, DHA, or ARA between APOE3 and APOE4 groups after 3 or 12 months of n-3 PUFA supplementation (Figures $2 \mathrm{~A}-\mathrm{C}$ ). However, higher concentrations of EPA and DHA-derived oxylipins were observed in the APOE4 group compared to the $A P O E 3$ group. Differences were observed at 12 months 
TABLE 3 | Baseline plasma phosphatidylcholine fatty acids (\% of total fatty acids; \%tFA) and oxylipin concentrations (nM) in APOE3 and APOE4 individuals.

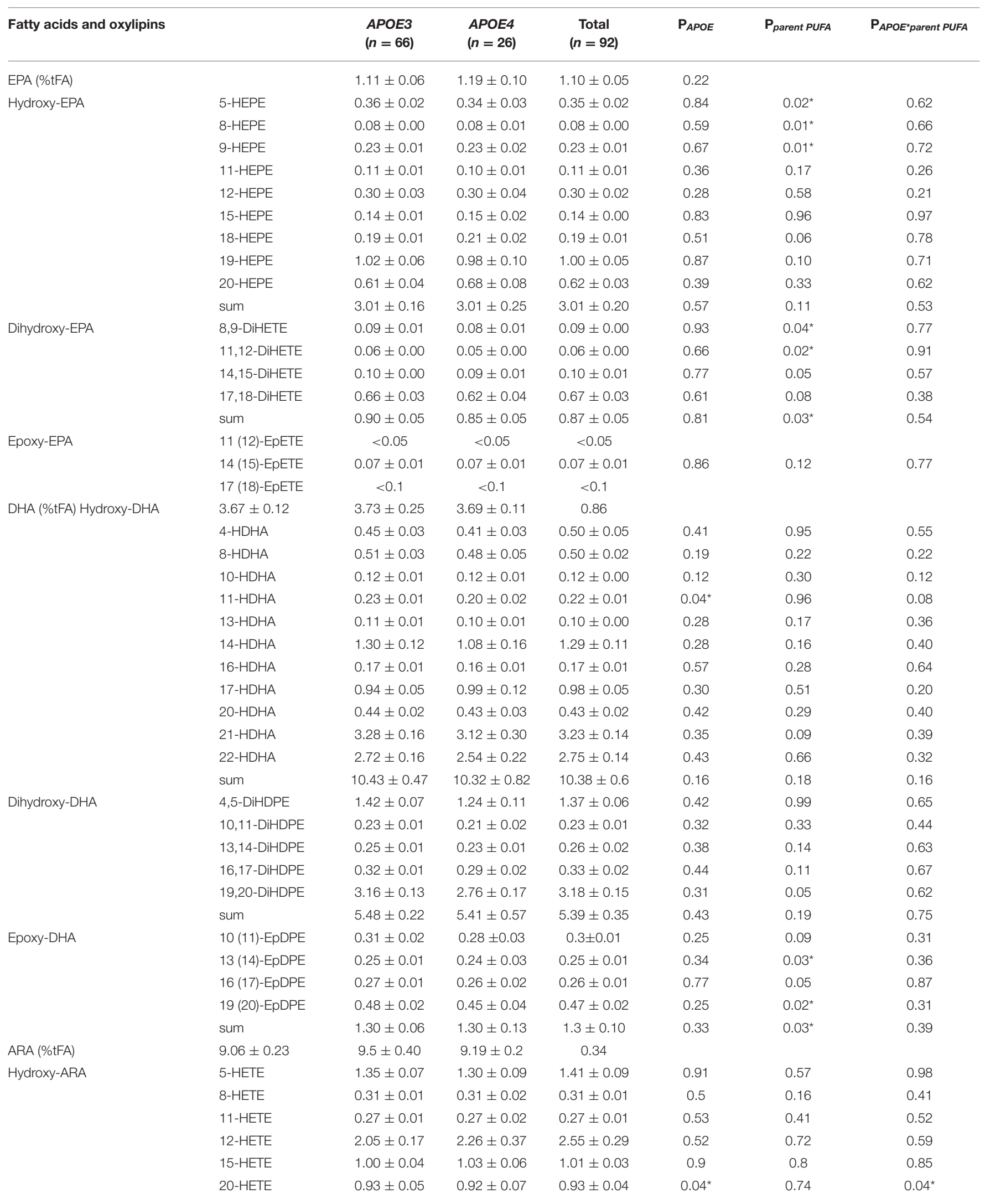


TABLE 3 | Continued

\begin{tabular}{|c|c|c|c|c|c|c|c|}
\hline Fatty acids and oxylipins & & $\begin{array}{l}\text { APOE3 } \\
(n=66)\end{array}$ & $\begin{array}{l}\text { APOE4 } \\
(n=26)\end{array}$ & $\begin{array}{c}\text { Total } \\
(n=92)\end{array}$ & $\mathbf{P}_{A P O E}$ & $\mathbf{P}_{\text {parent PUFA }}$ & $\mathbf{P}_{A P O E^{\star} \text { parent }}$ PUFA \\
\hline & sum & $6.21 \pm 0.12$ & $6.55 \pm 0.09$ & $6.39 \pm 0.10$ & 0.41 & 0.88 & 0.46 \\
\hline \multirow[t]{5}{*}{ Dihydroxy-ARA } & 5,6-DiHETrE & $0.37 \pm 0.04$ & $0.27 \pm 0.02$ & $0.34 \pm 0.03$ & 0.51 & 0.82 & 0.31 \\
\hline & 8,9-DiHETrE & $0.25 \pm 0.01$ & $0.21 \pm 0.01$ & $0.24 \pm 0.01$ & 0.42 & 0.20 & 0.22 \\
\hline & 11,12-DiHETrE & $0.57 \pm 0.02$ & $0.61 \pm 0.07$ & $0.58 \pm 0.02$ & 0.82 & 0.19 & 0.95 \\
\hline & 14,15-DiHETrE & $0.65 \pm 0.02$ & $0.70 \pm 0.07$ & $0.66 \pm 0.02$ & 0.62 & 0.19 & 0.76 \\
\hline & sum & $1.84 \pm 0.05$ & $1.68 \pm 0.06$ & $1.78 \pm 0.06$ & 0.46 & 0.36 & 0.32 \\
\hline \multirow[t]{5}{*}{ Epoxy-ARA } & 5 (6)-EpETrE & $0.9 \pm 0.05$ & $0.8 \pm 0.09$ & $0.87 \pm 0.04$ & 0.94 & 0.49 & 0.90 \\
\hline & 8 (9)-EpETrE & $0.19 \pm 0.01$ & $0.18 \pm 0.02$ & $0.19 \pm 0.01$ & 0.89 & 0.19 & 0.77 \\
\hline & 11 (12)-EpETrE & $0.20 \pm 0.01$ & $0.19 \pm 0.01$ & $0.20 \pm 0.01$ & 0.56 & 0.29 & 0.47 \\
\hline & 14 (15)-EpETrE & $0.46 \pm 0.02$ & $0.42 \pm 0.03$ & $0.45 \pm 0.02$ & 0.63 & 0.29 & 0.46 \\
\hline & sum & $1.75 \pm 0.22$ & $1.69 \pm 0.57$ & $1.73 \pm 0.37$ & 0.57 & 0.50 & 0.94 \\
\hline
\end{tabular}

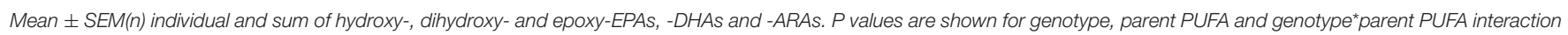
using a univariate GLM. Age, gender, and BMI were used as covariates. *Statistically significant, $P<0.05$.

(Figures 2D,E,G,H,J,K) for the sum of HEPEs, DiHETEs, EpETEs, HDHAs and DiHDPEs $(p=0.014, p=0.001, p=0.024$, $p=0.048$, and $p=0.011$, respectively). There was no significant difference between APOE3 and APOE4 in the concentrations of epoxy-DHAs (Figure 2K) or epoxy-ARAs (Figures 2F,I,L). Analysis of n-3 PUFA* APOE (independent and interactive) was carried out on select LA- ALA- and DGLA- oxylipins. There were no significant effects evident (data not shown).

Considering the change in the sum of oxylipins derived from EPA and DHA at 12 months, a linear dose-response increase in oxylipins was observed as described previously (22). For the hydroxy- and dihydroxy-EPAs and -DHAs, but not for the epoxy-EPAs and -DHAs, the increase was significantly greater in the APOE4 carriers who received the highest n-3 PUFA dose (equivalent to 4 portions of fatty fish/week) (Figure 3 ).

A focused analysis of the change in the parent PUFA and the corresponding oxylipins at the highest dose of n-3 PUFAs supplemented (equivalent to 4 portions of fatty fish/week) was done. Change in the parent EPA was higher $(359 \% \pm 32)$ than DHA $(192 \% \pm 14)$, with no difference observed between APOE3 and APOE4 groups (Figures 4A,B). The increase in EPA- and DHA-derived oxylipins was generally higher than their parent PUFA. A greater increase in almost all oxylipins was observed in the APOE4 group, with the highest \% change seen in the EPA-derived 8 -HEPE $(1,474 \%$ in E4 compared to $477 \%$ in E3) ( $p=0.014$ ) (Figure 4A). With regard to DHA-derived oxylipins, the highest \% change was seen for 10-HDHA (597\% in E4 compared to $274 \%$ in E3, $p=0.026$ ) (Figure 4B). There was no significant difference in the epoxy-EPAs and -DHAs between $A P O E 3$ and $A P O E 4$ groups. After adjusting for age, sex, BMI and the basal level of parent n-3 PUFA, significant genotype*dose interactions were observed for two EPA-derived oxylipins: 19HEPE and 20-HEPE ( $p=0.027$ and 0.011 (Table 4).

\section{Effect of APOE Genotype Is More Evident for the Dihydroxy-EPAs and -DHAs Compared to the Epoxy-EPAs and -DHAs}

A strong independent effect of APOE genotype on all dihydroxyEPAs and -DHAs (except 4,5 DiHDPE) was observed (Table 4).
Despite the higher levels of epoxy-EPAs and -DHAs in the APOE4 group, the differences were not statistically significant (Table 4 and Figures 4A,B).

\section{Influence of the Basal Parent Plasma PUFA on the Change in Plasma Oxylipins Is More Pronounced for EPA-Derived Oxylipins}

The basal level of plasma PC EPA had a significant effect on the change in concentration of select hydroxy- and dihydroxy-EPAs (Table 4) but not epoxy-EPAs. When dividing baseline plasma PC EPA levels into tertiles and different, a higher change in 5HEPE, 9-HEPE, 11-HEPE, and 20-HEPE was observed at a low basal EPA level (EPA $<0.85 \%$ of total fatty acids) compared to high basal EPA level (EPA $>1.22 \%$ of total fatty acids) in APOE4 carriers (Figure 5). On the other hand, the basal level of plasma PC DHA had no influence on the change in DHA-derived oxylipins (Table 4).

\section{DISCUSSION}

PUFAs mediate inflammatory status partly through the balance between n-6 PUFA-derived and n-3 PUFA-derived oxylipins (9). Recent studies show a linear response of EPA- and DHA-derived oxylipins to fish oil supplementation $(24,25)$. However, a strong inter-individual variation is observed. APOE genotype is known to modulate systemic inflammation and neuroinflammation, and the response to fish oil interventions, in healthy subjects (31) and in patients with cardiovascular (32) and cognitive disorders (33). To our knowledge, no previous studies have explored the effect of $A P O E$ genotype on oxylipins, either cross-sectionally or in response to n-3 PUFA supplementation. In the current study, we show that $A P O E$ genotype affects the plasma oxylipin concentrations and their response to EPA+DHA intervention in healthy participants. We observe higher levels of hydroxyand dihydroxy-EPA- and DHA-derived oxylipins in APOE4 carriers compared to the wild type $A P O E 3 / E 3$ genotype. This difference becomes more evident with higher doses of n-3 PUFAs supplemented for longer periods (12 months). The greatest increase was in 8-HEPE, an oxylipin formed by autoxidation. 


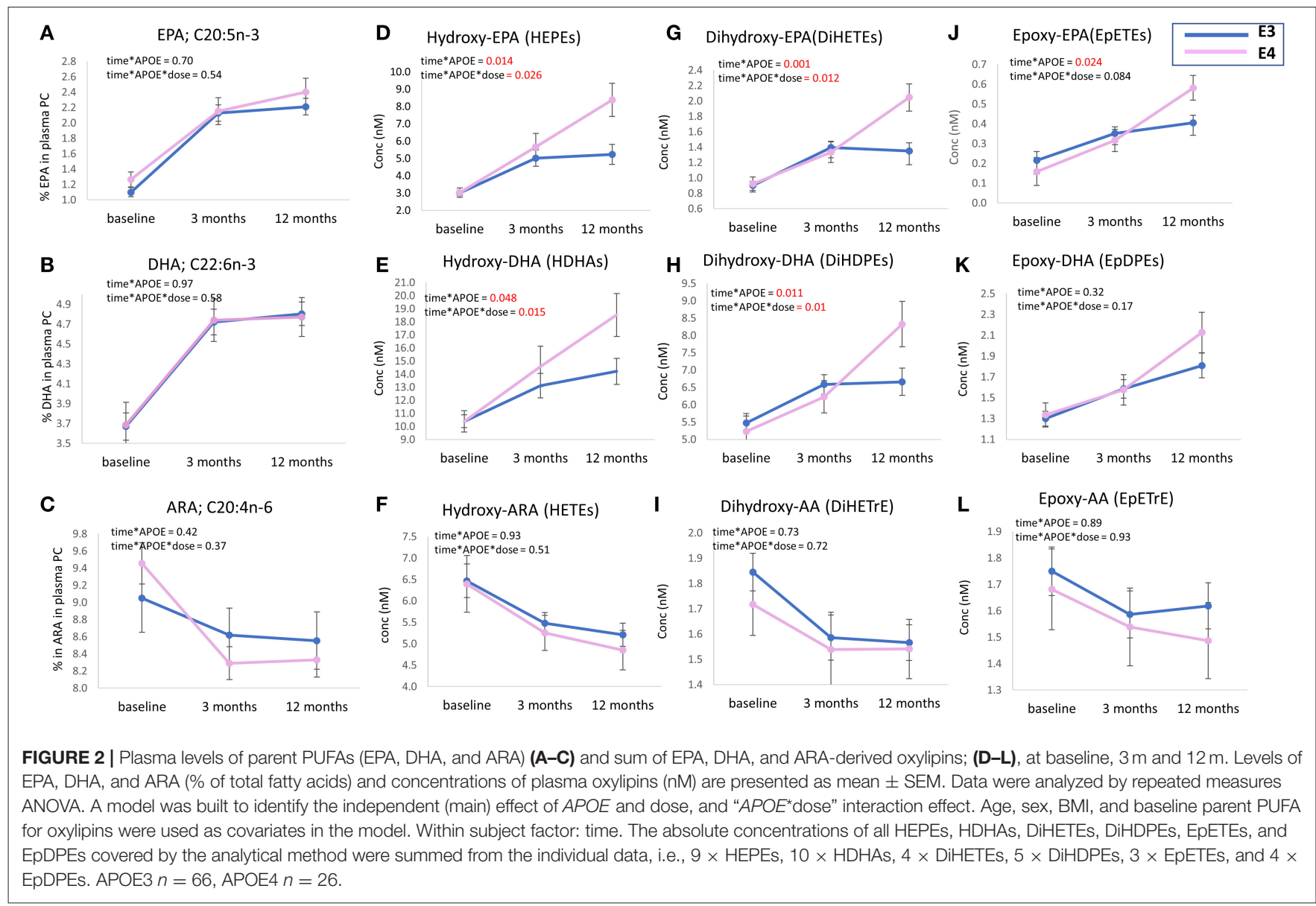

It has recently been shown that 8-HEPE, together with other HEPEs, has a high ligand activity for PPARs $(40,41)$. A significant effect of $A P O E$ on dihydroxy- but not the epoxy-EPAs and DHAs suggests a more active sEH enzyme in APOE4 carriers. Analyzing samples from a well-designed intervention trial, with different doses of n-3 PUFAs and for a duration up to 12 months, highlights the importance of n-3 PUFA dose and duration of intake in modulating select plasma oxylipin levels such as 8HEPE and 17-HDHA. Higher levels of these oxylipins in APOE4 carriers, may help mitigate a more disrupted metabolic and pro-inflammatory profile relative to the common $A P O E 3 / 3$ in multiple disease pathologies $(42,43)$.

Prior to intervention, there was no difference in the levels of ARA, EPA and DHA between APOE3 and APOE4 carriers (Table 3). This is consistent with the Multi-Ethnic Study of Atherosclerosis (MESA), where, although there was no difference in plasma phospholipid EPA and DHA concentrations between APOE 3 and APOE4 groups an APOE* $n-3$ PUFA interaction was evident with high density lipoprotein cholesterol and particle size (44). Similarly, in the Alzheimer's Disease Cooperative Study there were no differences between $A P O E$ genotypes for EPA and DHA in plasma phospholipids at baseline (45). In contrast, Plourde et al. showed that EPA and DHA were higher in APOE4 carriers, in plasma triglycerides while there was no differences in n-3 PUFAs between genotypes in the non-esterified fatty acid fraction, with plasma phospholipid fraction composition data not included (46).

Similar to baseline, there was no difference in the levels of EPA, DHA or ARA between APOE3 and APOE4 groups after 12 months of n-3 PUFA supplementation (Figures 2A-C), which is consistent with previous n-3 PUFA intervention studies carried out in healthy subjects $(47,48)$ and after 18 months of DHA supplementation in patients with Alzheimer's disease (49). However, in some studies, the ratios of DHA/AA and EPA/AA were lower in the APOE4 group following n-3 PUFA supplementation (45). This was not found in the current analysis (data not shown).

At baseline, there was no difference in plasma oxylipin levels between APOE3 and APOE4 except for 11-HDHA and 20HETE, which were both lower in the APOE4 group (Table 3 ). In mouse models, there was no significant difference in the level of 20-HETE between wild type and APOE knockout mice. However, after being fed with a high fat diet, 20-HETE was higher in the renal tissue of the APOE knockout mice, with no difference observed in the hepatic tissue (50). Similarly, in a mouse model of abdominal aortic aneurysm, levels of several HETEs (5-, 8-, 12-, 15-HETE) were similar in the blood of wildtype and $A P O E$ knockout mice, and were higher in 


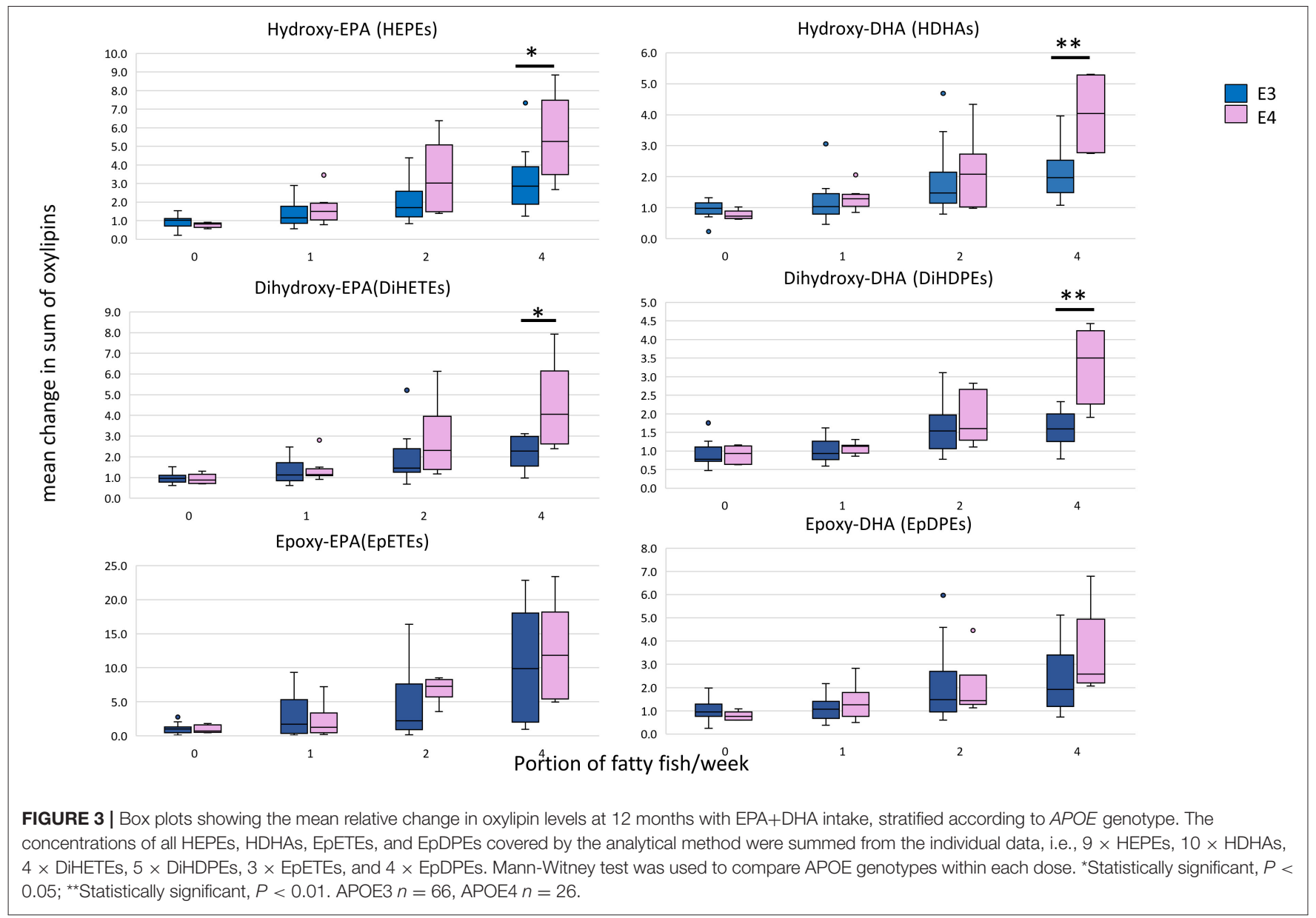

APOE knockout mice after pro-coagulant administration (51). In aged mice, brain cortical levels of EPA-derived 18-HEPE and DHA-derived 10,17-diHDHA, together with the specialized pro-resolving mediator resolvin $\mathrm{D} 1$, were lower in APOE4 mice compared to APOE3 (52).

The changes in plasma EPA- and DHA-oxylipins were consistently higher in APOE4 compared to APOE3/E3 (Figures 4A,B), with the highest change observed for 8HEPE $(1,474 \%$ in APOE4 vs. $477 \%$ in APOE $3, p=0.014)$ (Figure 4A). Most plasma oxylipins are bound to lipoproteins (53), and in particular LDL (54). APOE4 has a higher affinity for the LDL-receptor leading to increased catabolism of VLDL and a subsequent increase in LDL-cholesterol $(55,56)$. In addition, $n-3$ PUFA supplementation was found to increase LDL-cholesterol concentrations (57), which becomes more pronounced in APOE4 carriers, and more evident in chronic inflammatory conditions (32). Interestingly, after 1 year of $0.840 \mathrm{~g} /$ day EPA+DHA and vitamin $\mathrm{D}$ supplementation in a subset of the VITAL study, an association between some oxylipins and increased LDL was found (58). Consequently, although LDL data are not available in the current RCT, it is speculated that, with higher doses of EPA and DHA, the differential increase of oxylipins in APOE4 carriers could relate to possibly higher LDL levels in those individuals. However, given the healthy status of the participants in this study, future studies focusing on patients with chronic inflammatory conditions are needed for confirmation.

Recent studies show that HEPEs have higher ligand activity for PPARs than their parent EPA $(40,41)$. 8-HEPE activated the transcription of PPARs leading to increased adipogenesis and cellular glucose uptake in fibroblasts and muscle cell-lines (41), and improved dyslipidemia in a PPAR $\alpha$-dependent manner (17). Several studies showed that glucose and lipid metabolism and fatty acid oxidation are disturbed in APOE4 carriers (59-62). Interestingly, PPAR $\gamma$ signaling was also found to be disturbed in APOE4 carriers $(60,63,64)$. Taken together, we suggest that the post-n-3 PUFA intervention differential increase in levels of HEPEs observed here, especially in 8-HEPE, promotes PPAR $\gamma$ activation, and consequently could contribute to the partial mitigation of the disturbed metabolic processes evident in APOE4 carriers.

APOE4 individuals have a higher inflammatory status and more oxidative stress $(65,66)$ compared to APOE3 carriers. LPS-stimulated macrophages from human and mice showed increased TNF- $\alpha$ and IL- 6 , and the activation of the inflammatory NFKB pathway in APOE4 compared to APOE3 carriers (65). We have previously shown higher levels of h-CRP, P-selectin and E-selectin in normal-weight healthy APOE4 individuals in comparison to APOE3/E3 (67). Similarly, in the mouse brain, 


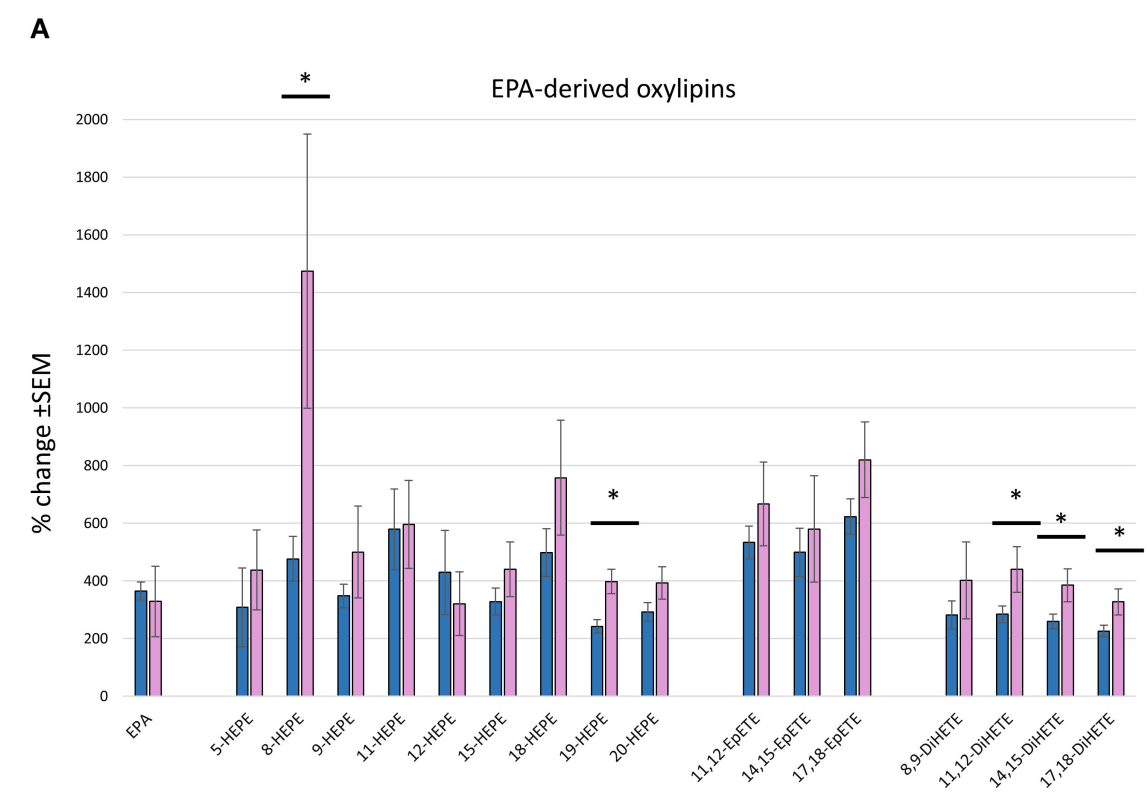

B DHA-derived oxylipins

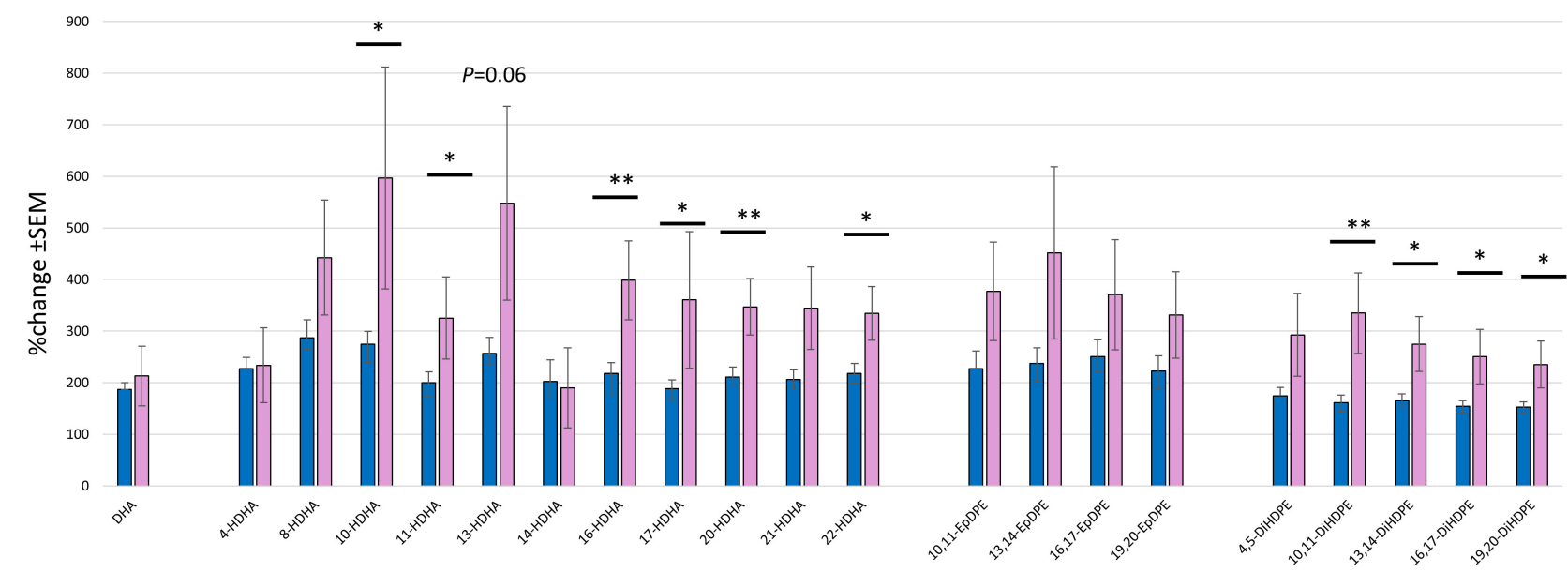

FIGURE 4 | Bar charts showing the mean \% change (with SEM) in individual EPA- (A) and DHA- (B) derived oxylipins, with their parent EPA and DHA following 12 months of supplementation with the equivalent of 4 portions of oily fish, comparing between APOE3 $(n=17)$ and APOE4 $(n=5 / 6)$. Independent sample $t$-test or Mann-Witney test was used to compare between $A P O E$ genotypes. ${ }^{*}$ Statistically significant, $P<0.05,{ }^{\star}$ Statistically significant, $P<0.01$.

an $A P O E 4$ genotype was associated with increased microglial activation, IL-1 $\beta$ and lipid peroxidation $(65,66)$.

A possible mechanism for the increase in HEPEs and HDHAs in APOE4 carriers could be the increased activity of 5- and 12/15-lipoxygenases and increased auto-oxidation in response to the higher inflammatory status in APOE4 carriers. Increased activity of lipoxygenase enzymes was found in the macrophages and atherosclerotic plaques of $A P O E$ KO mice used as models of chronic inflammation (68, 69). Moreover, the absence of 12/15-lipoxygenase reduced oxidative stress in the brains of APOE KO mice (70). N3 PUFA were shown to increase lipoxins and resolvins in atherosclerosis (71) and Alzheimer's disease (72), thus suggesting EPA/DHA-induced activation of 5- and 12/15-lipoxygenases. Similarly, products of n-3 PUFA autooxidation increase with n3 PUFA supplementation, with studies showing their beneficial effects on cardiovascular diseases (8). Interestingly, in this study the 15-lipoxygenase derived oxylipin 17-HDHA was significantly higher in APOE4 $\left(P_{\mathrm{APOE}}=0.03\right)$ (Table 4 and Figure 4B). 17-HDHA is the precursor of D-resolvins and protectins which possess strong pro-resolving and antiinflammatory properties and are dysregulated in several chronic inflammatory and neurodegenerative conditions $(17,52)$. Taken together, we suggest that the inflammatory environment in 
TABLE 4 | Change from baseline in plasma oxylipins (nM) after 12 months of supplementation with n-3 PUFAs equivalent to $0,1,2$, and 4 portions of fatty fish per week.

\begin{tabular}{|c|c|c|c|c|c|c|c|c|c|c|c|c|c|c|c|c|}
\hline \multirow[t]{2}{*}{ Oxylipin } & \multicolumn{12}{|c|}{ Portion of fish oil per week equivalency } & \multirow{2}{*}{$\mathrm{P}_{A P O E}$} & \multirow{2}{*}{$P_{\text {dose }}$} & \multirow{2}{*}{$\begin{array}{l}\mathrm{P}_{A P O E^{*}} \\
\text { dose }\end{array}$} & \multirow{2}{*}{$\begin{array}{c}\mathrm{P}_{\text {baselin }} \\
\text { parent } \\
\text { PUFA }\end{array}$} \\
\hline & $\begin{array}{l}A P O E 3 \\
(n=14)\end{array}$ & $\begin{array}{l}A P O E 4 \\
(n=5)\end{array}$ & $\begin{array}{c}\text { Total } \\
(n=19)\end{array}$ & $\begin{array}{l}\text { APOE3 } \\
(n=17)\end{array}$ & $\begin{array}{l}\text { APOE4 } \\
(n=10)\end{array}$ & $\begin{array}{c}\text { Total } \\
(n=27)\end{array}$ & $\begin{array}{l}\text { APOE3 } \\
(n=17)\end{array}$ & $\begin{array}{c}A P O E 4 \\
(n=6)\end{array}$ & $\begin{array}{c}\text { Total } \\
(n=23)\end{array}$ & $\begin{array}{l}A P O E 3 \\
(n=17)\end{array}$ & $\begin{array}{l}\text { APOE4 } \\
(n=5 / 6)\end{array}$ & $\begin{array}{c}\text { Total } \\
(n=22 / 23)\end{array}$ & & & & \\
\hline \multicolumn{17}{|l|}{ Hydroxy-EPA } \\
\hline 5-HEPE & $-0.04 \pm 0.03$ & $-0.08 \pm 0.05$ & $-0.05 \pm 0.03$ & $0.14 \pm 0.07$ & $0.12 \pm 0.08$ & $0.14 \pm 0.05$ & $0.25 \pm 0.06$ & $0.52 \pm 0.19$ & $0.32 \pm 0.07$ & $0.67 \pm 0.08$ & $0.57 \pm 0.17$ & $0.66 \pm 0.07$ & 0.463 & $<0.001$ & 0.117 & 0.001 \\
\hline 8-HEPE & $-0.02 \pm 0.02$ & $-0.01 \pm 0.01$ & $-0.02 \pm 0.01$ & $0.09 \pm 0.04$ & $31 \pm 0.20$ & $17 \pm 0.08$ & $12 \pm 0.04$ & $0.28 \pm 0.16$ & $0.16 \pm 0.05$ & $0.27 \pm 0.06$ & $0.70 \pm 0.08$ & $0.33 \pm 0.06$ & 0.004 & 0.001 & 0.486 & 0.104 \\
\hline 9-HEPE & $0.00 \pm 0.05$ & $-0.07 \pm 0.04$ & $-0.02 \pm 0.04$ & $14 \pm 0.06$ & $27 \pm 0.15$ & $19 \pm 0.06$ & $23 \pm 0.05$ & $0.50 \pm 0.18$ & $0.30 \pm 0.06$ & $0.55 \pm 0.07$ & $0.92 \pm 0.14$ & $0.60 \pm 0.07$ & 0.006 & $<0.001$ & 0.259 & 0.005 \\
\hline 11-HEPE & $-0.03 \pm 0.02$ & $-0.01 \pm 0.01$ & $-0.03 \pm 0.01$ & $0.11 \pm 0.03$ & $0.13 \pm 0.07$ & $0.12 \pm 0.03$ & $0.13 \pm 0.03$ & $0.29 \pm 0.14$ & $0.17 \pm 0.04$ & $0.30 \pm 0.05$ & $0.50 \pm 0.13$ & $0.33 \pm 0.05$ & 0.012 & $<0.001$ & 0.207 & 0.005 \\
\hline 12-HEPE & $-0.02 \pm 0.04$ & $-0.06 \pm 0.06$ & $-0.03 \pm 0.03$ & $0.04 \pm 0.09$ & $31 \pm 0.16$ & $.14 \pm 0.08$ & $0.38 \pm 0.13$ & $0.95 \pm 0.42$ & $0.53 \pm 0.15$ & $0.52 \pm 0.16$ & $0.27 \pm 0.22$ & $0.49 \pm 0.14$ & 0.256 & 0.002 & 0.196 & 0.114 \\
\hline 15-HEPE & $<0.13$ & $<0.13$ & $<0.13$ & $<0.13$ & $<0.13$ & $<0.13$ & $<0.13$ & $<0.13$ & $<0.13$ & $0.17 \pm 0.09$ & $0.33 \pm 0.33$ & $0.19 \pm 0.09$ & 0.527 & 0.022 & 0.385 & 0.356 \\
\hline 18-HEPE & $-0.01 \pm 0.02$ & $0.01 \pm 0.04$ & $0 \pm 0.01$ & $0.11 \pm 0.04$ & $0.71 \pm 0.42$ & $0.33 \pm 0.16$ & $0.2 \pm 0.04$ & $0.37 \pm 0.17$ & $0.24 \pm 0.05$ & $0.58 \pm 0.1$ & $1.32 \pm 0.56$ & $0.69 \pm 0.12$ & 0.008 & 0.001 & 0.265 & 0.767 \\
\hline 19-HEPE & $-0.05 \pm 0.12$ & $-0.13 \pm 0.07$ & $-0.07 \pm 0.09$ & $0.21 \pm 0.15$ & $17 \pm 0.09$ & $0.20 \pm 0.10$ & $0.7 \pm 0.22$ & $1.68 \pm 0.64$ & $0.96 \pm 0.24$ & $1.36 \pm 0.24$ & $2.02 \pm 0.2$ & $1.45 \pm 0.21$ & 0.026 & $<0.001$ & 0.027 & 0.024 \\
\hline 20-HEPE & $-0.05 \pm 0.09$ & $-0.29 \pm 0.17$ & $-0.11 \pm 0.08$ & $25 \pm 0.11$ & $0.11 \pm 0.07$ & $0.20 \pm 0.07$ & $0.42 \pm 0.11$ & $0.95 \pm 0.38$ & $0.56 \pm 0.13$ & $1.10 \pm 0.13$ & $1.33 \pm 0.09$ & $1.13 \pm 0.12$ & 0.198 & 0.001 & 0.011 & 0.008 \\
\hline \multicolumn{17}{|l|}{ Dihydroxy-EPA } \\
\hline 8,9-DiHETE & $-0.03 \pm 0.01$ & $\begin{array}{c}-0.00 \pm 0.01 \\
0\end{array}$ & $-0.02 \pm 0.01$ & $0.02 \pm 0.01$ & $77 \pm 0.03$ & $84 \pm 0.01$ & $04 \pm 0.02$ & $10 \pm 0.04$ & $0.06 \pm 0.02$ & $0.13 \pm 0.02$ & $.17 \pm 0.03$ & $0.14 \pm 0.02$ & 0.007 & 01 & 0.633 & 0.003 \\
\hline 11,12-DiHETE & $-0.01 \pm 0.01$ & $0.00 \pm 0.01$ & $-0.01 \pm 0.01$ & $0.01 \pm 0.01$ & $07 \pm 0.04$ & $03 \pm 0.02$ & $04 \pm 0.01$ & $0.07 \pm 0.02$ & $0.05 \pm 0.01$ & $0.09 \pm 0.01$ & $0.18 \pm 0.01$ & $0.10 \pm 0.01$ & 0.002 & $<0.001$ & 0.642 & 0.07 \\
\hline 14,15-DiHETE & $-0.01 \pm 0.01$ & $-0.01 \pm 0.01$ & $-0.01 \pm 0.01$ & $0.02 \pm 0.01$ & $0.08 \pm 0.05$ & $0.04 \pm 0.02$ & $0.07 \pm 0.02$ & $0.11 \pm 0.03$ & $0.08 \pm 0.01$ & $0.13 \pm 0.02$ & $0.26 \pm 0.03$ & $0.15 \pm 0.02$ & 0.001 & $<0.001$ & 0.360 & 0.023 \\
\hline 17,18-DiHETE & $-0.04 \pm 0.06$ & $-0.01 \pm 0.07$ & $-0.03 \pm 0.05$ & $5+009$ & $9+021$ & $24 \pm 0.10$ & $46 \pm 0.12$ & $0.83 \pm 0.25$ & $55+0.11$ & $072+01$ & $1.45 \pm 0.17$ & $0.83 \pm 0.10$ & 0.001 & & 0.236 & 04 \\
\hline \multicolumn{17}{|l|}{ Epoxy-EPA } \\
\hline 11 (12)-EpETE & $<0.05$ & $<0.05$ & $<0.05$ & $0.03 \pm 0.01$ & $0.02 \pm 0.01$ & 0.030 & $0.06 \pm 0.01$ & $0.07 \pm 0.02$ & $0.06 \pm 0.01$ & $0.13 \pm 0.02$ & $0.17 \pm 0.04$ & $0.14 \pm 0.02$ & 0.605 & $<0.001$ & 0.302 & 0.343 \\
\hline 14 (15)-EpETE & $0 \pm 0.01$ & $-0.01 \pm 0.01$ & $0 \pm 0.01$ & $0.02 \pm 0.01$ & $0.02 \pm 0.02$ & $02 \pm 0.01$ & $0.09 \pm 0.02$ & $0.11 \pm 0.04$ & $0.09 \pm 0.02$ & $0.17 \pm 0.03$ & $0.23 \pm 0.04$ & $0.18 \pm 0.02$ & 0.450 & $<0.001$ & 0.818 & 0.419 \\
\hline 17 (18)-EpETE & $<0.1$ & $<0.1$ & $<0.1$ & $<0.1$ & $<0.1$ & $<0.1$ & $4 \pm 0.03$ & $0.21 \pm 0.06$ & $0.16 \pm 0.03$ & $0.26 \pm 0.03$ & $0.36 \pm 0.07$ & $0.28 \pm 0.03$ & 0.067 & & 0.294 & 0.213 \\
\hline \multicolumn{17}{|l|}{ Hydroxy-DHA } \\
\hline 4-HDHA & $-0.08 \pm 0.07$ & $0.01 \pm 0.14$ & $-0.05 \pm 0.06$ & $0.06 \pm 0.07$ & $0.14 \pm 0.09$ & $0.09 \pm 0.06$ & $0.26 \pm 0.08$ & $0.36 \pm 0.12$ & $0.29 \pm 0.07$ & $0.48 \pm 0.06$ & $0.45 \pm 0.17$ & $0.47 \pm 0.05$ & 0.458 & $<0.001$ & 0.994 & 0.688 \\
\hline 7-HDHA & $<0.1$ & $<0.1$ & $<0.1$ & $0.13 \pm 0.02$ & $0.11 \pm 0.02$ & $0.12 \pm 0.02$ & $0.16 \pm 0.02$ & $0.23 \pm 0.09$ & $0.18 \pm 0.03$ & $0.25 \pm 0.03$ & $0.31 \pm 0.06$ & $0.26 \pm 0.03$ & 0.301 & $<0.001$ & 0.54 & 0.605 \\
\hline 8-HDHA & $-0.08 \pm 0.09$ & $-0.15 \pm 0.12$ & $-0.1 \pm 0.07$ & $0.21 \pm 0.09$ & $0.28 \pm 0.11$ & $0.24 \pm 0.07$ & $0.37 \pm 0.09$ & $0.68 \pm 0.31$ & $0.45 \pm 0.10$ & $0.82 \pm 0.12$ & $1.34 \pm 0.21$ & $0.90 \pm 0.11$ & 0.062 & $<0.001$ & 0.458 & 0.514 \\
\hline 10-HDHA & $-0.02 \pm 0.01$ & $-0.01 \pm 0.03$ & $-0.02 \pm 0.01$ & $0.03 \pm 0.02$ & $0.11 \pm 0.04$ & $0.06 \pm 0.02$ & $0.1 \pm 0.02$ & $0.19 \pm 0.10$ & $0.12 \pm 0.03$ & $0.19 \pm 0.02$ & $0.35 \pm 0.02$ & $0.21 \pm 0.02$ & 0.008 & $<0.001$ & .577 & 1.000 \\
\hline 11-HDHA & $-0.03 \pm 0.02$ & $-0.02 \pm 0.05$ & $-0.03 \pm 0.02$ & $0.01 \pm 0.03$ & $0.14 \pm 0.04$ & $0.06 \pm 0.03$ & $0.15 \pm 0.04$ & $0.27 \pm 0.10$ & $0.18 \pm 0.04$ & $0.21 \pm 0.04$ & $0.34 \pm 0.01$ & $0.23 \pm 0.03$ & 0.016 & $<0.001$ & .658 & 0.717 \\
\hline 13-HDHA & $-0.01 \pm 0.01$ & $0.01 \pm 0.03$ & $-0.01 \pm 0.01$ & $0.04 \pm 0.02$ & $0.05 \pm 0.02$ & $0.05 \pm 0.02$ & $0.08 \pm 0.02$ & $0.14 \pm 0.09$ & $0.10 \pm 0.03$ & $0.15 \pm 0.02$ & $0.31 \pm 0.09$ & $0.17 \pm 0.03$ & 0.024 & $<0.001$ & 0.352 & 0.369 \\
\hline 14-HDHA & $0.09 \pm 0.16$ & $-0.42 \pm 0.43$ & $-0.04 \pm 0.16$ & $-0.38 \pm 0.32$ & $1.45 \pm 0.91$ & $0.3 \pm 0.42$ & $1.04 \pm 0.45$ & $2.7 \pm 1.35$ & $1.47 \pm 0.49$ & $0.61 \pm 0.34$ & $0.32 \pm 0.82$ & $0.57 \pm 0.31$ & 0.21 & 0.015 & 0.096 & 0.106 \\
\hline 16-HDHA & $-0.02 \pm 0.01$ & $-0.01 \pm 0.04$ & $-0.02 \pm 0.01$ & $0.05 \pm 0.02$ & $0.14 \pm 0.06$ & $0.08 \pm 0.03$ & $0.10 \pm 0.03$ & $0.17 \pm 0.09$ & $0.12 \pm 0.03$ & $0.18 \pm 0.03$ & $0.41 \pm 0.04$ & $0.22 \pm 0.03$ & 0.003 & $<0.001$ & .312 & 0.412 \\
\hline 17-HDHA & $-0.11 \pm 0.07$ & $-0.06 \pm 0.17$ & $-0.1 \pm 0.06$ & $0.06 \pm 0.15$ & $0.03 \pm 0.18$ & $0.05 \pm 0.11$ & $0.28 \pm 0.11$ & $0.67 \pm 0.22$ & $0.38 \pm 0.10$ & $0.63 \pm 0.11$ & $1.36 \pm 0.08$ & $0.73 \pm 0.11$ & 0.033 & $<0.001$ & .155 & 0.455 \\
\hline 20-HDHA & $-0.06 \pm 0.04$ & $0.02 \pm 0.13$ & $-0.04 \pm 0.04$ & $0.09 \pm 0.06$ & $0.35 \pm 0.17$ & $0.18 \pm 0.08$ & $0.24 \pm 0.05$ & $0.38 \pm 0.22$ & $0.28 \pm 0.07$ & $0.43 \pm 0.06$ & $0.93 \pm 0.10$ & $0.50 \pm 0.07$ & 0.004 & $<0.001$ & .429 & 0.666 \\
\hline 21-HDHA & $-0.10 \pm 0.48$ & $-1.08 \pm 0.37$ & $-0.36 \pm 0.38$ & $0.18 \pm 0.33$ & $0.26 \pm 0.34$ & $0.21 \pm 0.24$ & $1.62 \pm 0.38$ & $2.40 \pm 1.13$ & $1.82 \pm 0.40$ & $3.21 \pm 0.47$ & $4.68 \pm 0.63$ & $3.42 \pm 0.42$ & 0.357 & $<0.001$ & .434 & 0.093 \\
\hline 22-HDHA & $-0.10 \pm 0.41$ & $0.03 \pm 1.14$ & $-0.07 \pm 0.41$ & $0.08 \pm 0.35$ & $0.12 \pm 0.41$ & $0.1 \pm 0.26$ & $1.29 \pm 0.32$ & $1.99 \pm 0.97$ & $1.47 \pm 0.34$ & $3.08 \pm 0.48$ & $4.08 \pm 0.47$ & $3.23 \pm 0.42$ & 0.209 & $<0.001$ & 0.786 & 0.225 \\
\hline \multicolumn{17}{|l|}{ Dihydroxy-DHA } \\
\hline 4,5-DiHDPE & $-0.23 \pm 0.15$ & $-0.19 \pm 0.22$ & $-0.22 \pm 0.12$ & $0.05 \pm 0.16$ & $0 \pm 0.1$ & $6 \pm 0.11$ & $92 \pm 0.34$ & $1.15 \pm 0.27$ & $98 \pm 0.26$ & $0.80 \pm 0.19$ & $1.81 \pm 0.57$ & $0.95 \pm 0.19$ & 0.144 & 0.001 & .523 & 0.316 \\
\hline 10,11-DiHDPE & $-0.04 \pm 0.02$ & $-0.03 \pm 0.04$ & $-0.04 \pm 0.02$ & $0.00 \pm 0.03$ & $0.03 \pm 0.02$ & $0.01 \pm 0.02$ & $0.09 \pm 0.03$ & $0.18 \pm 0.10$ & $0.11 \pm 0.03$ & $0.12 \pm 0.03$ & $0.33 \pm 0.07$ & $0.15 \pm 0.03$ & 0.005 & $=0.001$ & 0.228 & 0.089 \\
\hline 13,14-DiHDPE & $-0.04 \pm 0.02$ & $-0.04 \pm 0.03$ & $-0.04 \pm 0.02$ & $0.01 \pm 0.02$ & $0.16 \pm 0.12$ & $0.06 \pm 0.05$ & $0.11 \pm 0.03$ & $0.14 \pm 0.05$ & $0.12 \pm 0.03$ & $0.14 \pm 0.03$ & $0.41 \pm 0.05$ & $0.18 \pm 0.03$ & 0.004 & $<0.001$ & 0.182 & 0.145 \\
\hline 16,17-DiHDPE & $-0.02 \pm 0.02$ & $-0.03 \pm 0.03$ & $-0.02 \pm 0.02$ & $-0.01 \pm 0.02$ & $0.16 \pm 0.11$ & $0.05 \pm 0.04$ & $0.16 \pm 0.04$ & $0.16 \pm 0.07$ & $0.16 \pm 0.03$ & $0.15 \pm 0.03$ & $0.42 \pm 0.07$ & $0.19 \pm 0.04$ & 0.008 & $<0.001$ & 0.100 & 0.095 \\
\hline 19,20-DiHDPE & $-0.44 \pm 0.29$ & $-0.25 \pm 0.32$ & $-0.39 \pm 0.22$ & $0.02 \pm 0.21$ & $1.33 \pm 1.10$ & $0.51 \pm 0.43$ & $1.45 \pm 0.37$ & $1.72 \pm 0.68$ & $1.52 \pm 0.32$ & $1.39 \pm 0.28$ & $3.85 \pm 0.77$ & $1.74 \pm 0.32$ & 0.006 & $<0.001$ & 0.349 & 0.054 \\
\hline
\end{tabular}




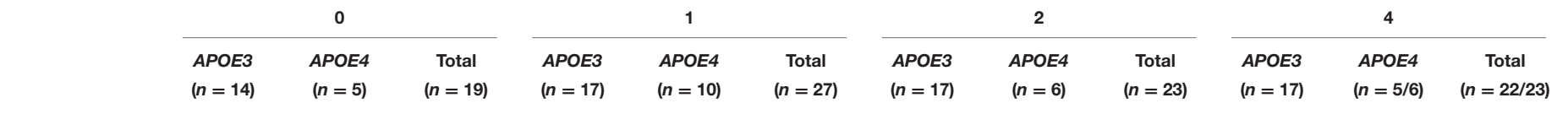

Epoxy-DHA

10 (11)-EpDPE

$\begin{array}{lllllllllllllll}0.00 \pm 0.04 & -0.05 \pm 0.03 & -0.01 \pm 0.03 & 0.00 \pm 0.04 & 0.03 \pm 0.05 & 0.02 \pm 0.03 & 0.16 \pm 0.06 & 0.24 \pm 0.10 & 0.18 \pm 0.05 & 0.31 \pm 0.09 & 0.43 \pm 0.09 & 0.32 \pm 0.07 & 0.496\end{array}$

$13(14)-E p D P E$

19 (20)-EpDPE

Hydroxy-ARA

5-HETE

8-HETE

11-HETE

12-HETE

15-HETE

20-HETE

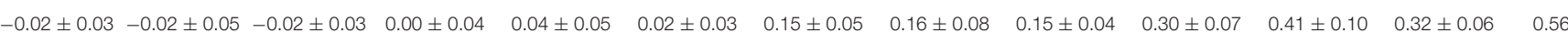
$\begin{array}{lllllllllllll}-0.01 \pm 0.05 & -0.10 \pm 0.04 & -0.03 \pm 0.04 & 0.02 \pm 0.06 & 0.07 \pm 0.06 & 0.04 \pm 0.04 & 0.25 \pm 0.08 & 0.37 \pm 0.19 & 0.29 \pm 0.08 & 0.47 \pm 0.11 & 0.69 \pm 0.10 & 0.50 \pm 0.10 & 0.405\end{array}$

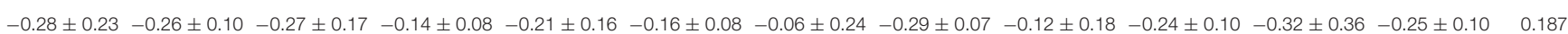

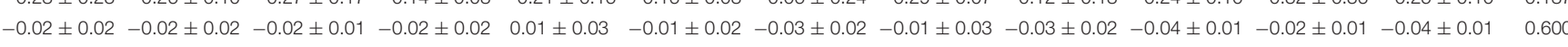
$\begin{array}{llllllllllllll}-0.03 \pm 0.01 & -0.07 \pm 0.02 & -0.04 \pm 0.01 & 0.00 \pm 0.02 & 0.00 \pm 0.05 & 0.00 \pm 0.02 & -0.04 \pm 0.03 & -0.02 \pm 0.02 & -0.04 \pm 0.02 & -0.06 \pm 0.01 & -0.05 \pm 0.03 & -0.06 \pm 0.01 & 0.545\end{array}$

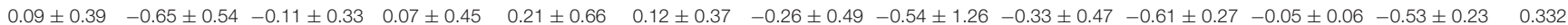

Dihydroxy-ARA

5,6-DiHETrE

8,9-DiHETrE

11,12-DiHETrE $0.04 \pm 0.09 \quad-0.08 \pm 0.14 \quad 0.01 \pm 0.08 \quad-0.10 \pm 0.08 \quad-0.25 \pm 0.08 \quad-0.16 \pm 0.06 \quad-0.18 \pm 0.05 \quad 0.10 \pm 0.18 \quad-0.10 \pm 0.06 \quad-0.22 \pm 0.08 \quad 0.15 \pm 0.22 \quad-0.17 \pm 0.08 \quad 0.827$

\section{Epoxy-ARA}

$\begin{array}{llllllllllllll}-0.18 \pm 0.15 & -0.02 \pm 0.01 & -0.14 \pm 0.11 & -0.02 \pm 0.03 & -0.01 \pm 0.03 & -0.02 \pm 0.02 & 0.02 \pm 0.15 & 0 \pm 0.04 & 0.02 \pm 0.11 & -0.08 \pm 0.04 & -0.08 \pm 0.1 & -0.08 \pm 0.04 & 0.544\end{array}$

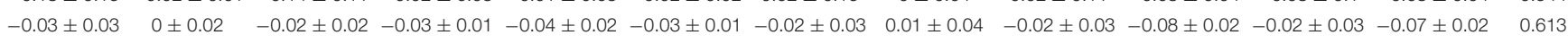

5 (6)-EpETrE $-0.04 \pm 0.03-0.03 \pm 0.04-0.04 \pm 0.03 \quad-0.06 \pm 0.02-0.16 \pm 0.03 \quad-0.1 \pm 0.02 \quad-0.08 \pm 0.03 \quad-0.04 \pm 0.04-0.07 \pm 0.02 \quad-0.19 \pm 0.03 \quad-0.01 \pm 0.08-0.17 \pm 0.03 \quad 0.369$

8 (9)-EpETrE

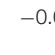

11 (12)-EpETrE

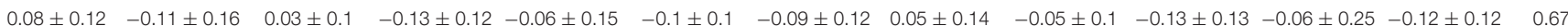
$\begin{array}{lllllllllllll}0 \pm 0.02 & 0 \pm 0.04 & 0 \pm 0.02 & -0.02 \pm 0.02 & 0 \pm 0.03 & -0.01 \pm 0.02 & -0.01 \pm 0.03 & 0.01 \pm 0.04 & 0 \pm 0.02 & 0 \pm 0.02 & 0 \pm 0.03 & 0 \pm 0.02 & 0.736\end{array}$ 


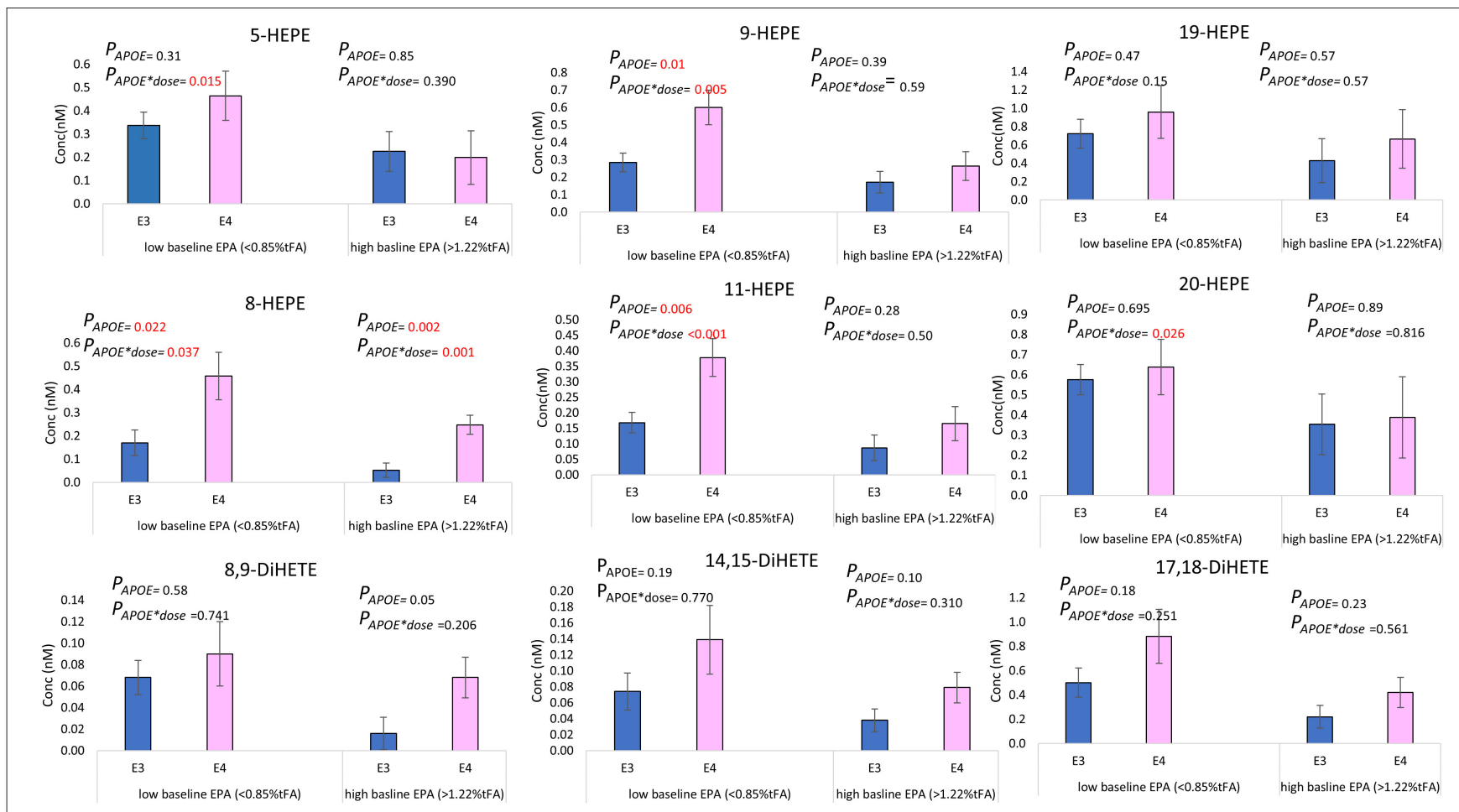

FIGURE 5 | Mean absolute change ( \pm SEM) in select EPA-derived oxylipins (nM) at 12 months of supplementation, according to $A P O E$ genotype and the level of baseline EPA. Low basal EPA (APOE3 $n=22$, APOE4 $n=9$ ), high basal EPA (APOE3 $n=18$, APOE4 $n=10)$.

APOE4 carriers could increase the activity of lipoxygenases and autooxidation of PUFA which in turn leads to an increased production of EPA-and DHA-oxylipins when fish oil is supplemented.

Consistent with previous observations of greater increases in oxylipins in those with lower parent PUFA at baseline $(25,73)$, we observed that the change in the EPA- oxylipins (5-, 9-, 11-, 19, 20HEPEs-, and all DiHETEs), though not the DHA- oxylipins, was associated with baseline EPA levels in plasma PC. In an earlier study, we found that the increase in 5-HEPE and 17,18-DiHETE after 12 weeks of $n-3$ PUFA supplementation was significantly associated with baseline EPA status $(p<0.01$ and $p<0.05$, respectively), with higher levels observed in subjects with low baseline EPA levels (23). The current study shows that higher levels of 5-HEPE, 9-HEPE, 11-HEPE, and 20-HEPE are present in APOE4 individuals with low basal EPA status, compared to $A P O E 3$ (Figure 5).

$A P O E^{*}$ time interactions were only evident for hydroxy- and dihydroxy-EPAs and -DHAs, with no significant increase in the epoxy-EPAs and DHAs (Figure 2 and Table 4). This is despite the increase of hydroxy-, dihydroxy- and epoxy-EPAs and -DHAs which was generally observed with n-3 PUFA intervention (Figure 2), Dihydroxy-oxylipins are the metabolic products of epoxy-oxylipins by the action of sEH, with the ratio of DiHETE/EpETE being indicative of sEH activity $(13,74)$.

It is important to note here the effect of n-3 PUFA dose and duration of intake on the changes in plasma oxylipins in
APOE4 carriers. The difference in n-3 PUFA-derived oxylipins was observed with an n-3 PUFA dose equivalent to two portions of oily fish intake per week and became more evident with the dose equivalent to four portions per week, at 12 months of n-3 PUFA supplementation. Indeed, it has been demonstrated that a threshold intake of n-3 PUFAs may be required before a favorable effect is observed, whether in the form of increased specialized pro-resolving mediators production (75) or in reducing neuroinflammation (76).

The main strengths of this study were the large number of oxylipins quantified, the dose response nature of the analysis (three physiologically relevant doses included), and the long intervention period of 12 months, which included interim assessment at 3 months.

The present study has some limitations. Due to the exploratory nature of the analysis, participants were genotyped retrospectively and an unequal sample size between APOE3 $(n=66)$ and APOE4 $(n=26)$ inevitable. When further subgrouping the participants according to n-3 PUFA dose, the numbers in the genotype groups were lower, reaching 5-6 in the four portions fatty fish/week group. However, an independent association of $A P O E$ with plasma oxylipins was still observed, regardless of the n-3 PUFA dose given. Another limitation is that correction for multiple testing was not applied, which may lead to overestimation of the findings. Due to the exploratory nature of this study, 
validation in a study with a larger number of participants is necessary.

In conclusion, this study shows for the first time the impact of $A P O E$ genotype on plasma oxylipin concentrations and their response to EPA+DHA intervention. Higher levels of EPA- and DHA- oxylipins in APOE4 carriers compared to the wild type $A P O E 3 / E 3$ genotype become more evident with higher doses of n-3 PUFAs supplemented for longer periods (12 months). The greatest increase was in autoxidatively formed 8-HEPE which is a PPAR activator, with PPAR activation shown previously to be inhibited in APOE4 carriers (64). This study indicates that $A P O E$ genotype mediating oxylipin production, and may be an important contributor to the inter-individual variability and dose-response relationship between fish oil supplementation and health outcomes. Future studies should focus on the HEPEs-PPARs-glucose and lipid metabolism axis according to APOE genotype status, given their greater increase in response to PUFA supplementation in APOE4 carriers and their emerging importance in regulating cellular metabolism.

\section{DATA AVAILABILITY STATEMENT}

The original contributions presented in the study are included in the article, further inquiries can be directed to the corresponding authors.

\section{REFERENCES}

1. Calder PC. Very long-chain n-3 fatty acids and human health: fact, fiction and the future. Proc Nutr Soc. (2018) 77:52-72. doi: 10.1017/S0029665117003950

2. Innes JK, Calder PC. Marine omega-3 (N-3) fatty acids for cardiovascular health: an update for 2020. Int J Mol Sci. (2020) 21:1362. doi: 10.3390/ijms21041362

3. Alexander DD, Miller PE, Van Elswyk ME, Kuratko CN, Bylsma LC. A Meta-analysis of randomized controlled trials and prospective cohort studies of eicosapentaenoic and docosahexaenoic long-chain omega-3 fatty acids and coronary heart disease risk. Mayo Clin Proc. (2017) 92:1529. doi: 10.1016/j.mayocp.2016.10.018

4. Wu S, Ding Y, Wu F, Li R, Hou J, Mao P. Omega-3 fatty acids intake and risks of dementia and Alzheimer's disease: a meta-analysis. Neurosci Biobehav Rev. (2015) 48:1-9. doi: 10.1016/j.neubiorev.2014.11.008

5. Gioxari A, Kaliora AC, Marantidou F, Panagiotakos DP. Intake of $\omega-3$ polyunsaturated fatty acids in patients with rheumatoid arthritis: a systematic review and meta-analysis. Nutrition. (2018) 45:114-24.e4. doi: 10.1016/j.nut.2017.06.023

6. D'Angelo S, Motti ML, Meccariello R. $\omega-3$ and $\omega-6$ polyunsaturated fatty acids, obesity and cancer. Nutrients. (2020) 12:2751. doi: 10.3390/nu12092751

7. Zhang Y, Zhuang P, He W, Chen JN, Wang WQ, Freedman ND, et al. Association of fish and long-chain omega-3 fatty acids intakes with total and cause-specific mortality: prospective analysis of 421309 individuals. J Intern Med. (2018) 284:399-417. doi: 10.1111/joim.12786

8. Anderson EJ, Taylor DA. Stressing the heart of the matter: re-thinking the mechanisms underlying therapeutic effects of n-3 polyunsaturated fatty acids. F1000 Med Rep. (2012) 4:13. doi: 10.3410/M4-13

9. Gabbs M, Leng S, Devassy JG, Monirujjaman M, Aukema HM. Advances in our understanding of oxylipins derived from dietary PUFAs. Adv Nutr. (2015) 6:513-40. doi: 10.3945/an.114.007732

\section{ETHICS STATEMENT}

The studies involving human participants were reviewed and approved by Suffolk Local Research Ethics Committee. The patients/participants provided their written informed consent to participate in this study.

\section{AUTHOR CONTRIBUTIONS}

PC and AW designed and carried out the original intervention study and conducted fatty acid analysis. $\mathrm{AO}$ and NS conducted the oxylipin measurements. RS and AM designed the current study, analyzed the data and performed statistical analysis, drafted the manuscript, and have primary responsibility for its final content. All authors read and approved the final manuscript.

\section{FUNDING}

This clinical trial was funded by the UK Foods Standards Agency (grants N05065 and N05066) and the UK Medical Research Council (grants U105960389 and U1052.00.014). Oxylipin measurements were supported by the German Research Foundation (grant Schebb 1801). APOE genotyping and the current data analysis were funded by EU-JPI/BBSRC (BB/P028233/1). We acknowledge Susan Jebb, Celia Walker and Lucy Browning for their contribution to the clinical trial.

10. Ishihara T, Yoshida M, Arita M. Omega-3 fatty acid-derived mediators that control inflammation and tissue homeostasis. Int Immunol. (2019) 31:55967. doi: 10.1093/intimm/dxz001

11. Nayeem MA. Role of oxylipins in cardiovascular diseases. Acta Pharmacol Sin. (2018) 39:1142-54. doi: 10.1038/aps.2018.24

12. Schunck W-H, Konkel A, Fischer R, Weylandt K-H. Therapeutic potential of omega-3 fatty acid-derived epoxyeicosanoids in cardiovascular and inflammatory diseases. Pharmacol Ther. (2018) 183:177-204. doi: 10.1016/j.pharmthera.2017. 10.016

13. Shahabi P, Siest G, Meyer UA, Visvikis-Siest S. Human cytochrome P450 epoxygenases: variability in expression and role in inflammation-related disorders. Pharmacol Ther. (2014) 144:134-61. doi: 10.1016/j.pharmthera.2014. 05.011

14. Arnold C, Markovic M, Blossey K, Wallukat G, Fischer R, Dechend R, et al. Arachidonic acid-metabolizing cytochrome P450 enzymes are targets of \{omega\}-3 fatty acids. J Biol Chem. (2010) 285:32720-33. doi: 10.1074/jbc.M110.118406

15. Zhang G, Panigrahy D, Mahakian LM, Yang J, Liu J-Y, Stephen Lee KS, et al. Epoxy metabolites of docosahexaenoic acid (DHA) inhibit angiogenesis, tumor growth, and metastasis. Proc Natl Acad Sci USA. (2013) 110:65305. doi: 10.1073/pnas.1304321110

16. Wang Y-XJ, Ulu A, Zhang L-N, Hammock B. Soluble epoxide hydrolase in atherosclerosis. Curr Atheroscler Rep. (2010) 12:174-83. doi: 10.1007/s11883-010-0108-5

17. Saito M, Ishida N, Yamada H, Ibi M, Hirose M. 8-HEPE-concentrated materials from pacific krill improve plasma cholesterol levels and hepatic steatosis in high cholesterol diet-fed low-density lipoprotein (LDL) receptor-deficient mice. Biol Pharm Bull. (2020) 43:919-24. doi: 10.1248/bpb.b20-00162 
18. Buckley CD, Gilroy DW, Serhan CN. Proresolving lipid mediators and mechanisms in the resolution of acute inflammation. Immunity. (2014) 40:315-27. doi: 10.1016/j.immuni.2014.02.009

19. Schuchardt JP, Ostermann AI, Stork L, Fritzsch S, Kohrs H, Greupner T, et al. Effect of DHA supplementation on oxylipin levels in plasma and immune cell stimulated blood. Prostaglandins Leukot Essent Fatty Acids. (2017) 121:7687. doi: 10.1016/j.plefa.2017.06.007

20. Fischer R, Konkel A, Mehling H, Blossey K, Gapelyuk A, Wessel N, et al. Dietary omega-3 fatty acids modulate the eicosanoid profile in man primarily via the CYP-epoxygenase pathway[S]. J Lipid Res. (2014) 55:115064. doi: 10.1194/jlr.M047357

21. Schuchardt JP, Ostermann AI, Stork L, Kutzner L, Kohrs H, Greupner T, et al. Effects of docosahexaenoic acid supplementation on PUFA levels in red blood cells and plasma. Prostaglandins Leukot Essent Fatty Acids. (2016) 115:12-23. doi: 10.1016/j.plefa.2016.10.005

22. Schebb NH, Ostermann AI, Yang J, Hammock BD, Hahn A, Schuchardt JP. Comparison of the effects of long-chain omega-3 fatty acid supplementation on plasma levels of free and esterified oxylipins. Prostaglandins Other Lipid Mediat. (2014) 113-115:21-9. doi: 10.1016/j.prostaglandins.2014.05.002

23. Schuchardt JP, Schmidt S, Kressel G, Willenberg I, Hammock BD, Hahn A, et al. Modulation of blood oxylipin levels by long-chain omega-3 fatty acid supplementation in hyper- and normolipidemic men. Prostaglandins Leukot Essent Fatty Acids. (2014) 90:27-37. doi: 10.1016/j.plefa.2013.12.008

24. Ostermann AI, West AL, Schoenfeld K, Browning LM, Walker CG, Jebb $\mathrm{SA}$, et al. Plasma oxylipins respond in a linear dose-response manner with increased intake of EPA and DHA: results from a randomized controlled trial in healthy humans. Am J Clin Nutr. (2019) 109:125163. doi: 10.1093/ajen/nqz016

25. Ostermann AI, Schebb NH. Effects of omega-3 fatty acid supplementation on the pattern of oxylipins: a short review about the modulation of hydroxy-, dihydroxy-, and epoxy-fatty acids. Food Funct. (2017) 8:235567. doi: 10.1039/C7FO00403F

26. Gladine C, Ostermann AI, Newman JW, Schebb NH. MS-based targeted metabolomics of eicosanoids and other oxylipins: analytical and inter-individual variabilities. Free Radic Biol Med. (2019) 144:72-89. doi: 10.1016/j.freeradbiomed.2019.05.012

27. Zhao J, Roman MJ, Devereux RB, Yeh F, Zhang Y, Haack K, et al. Leukotriene haplotype $\times$ diet interaction on carotid artery hypertrophy and atherosclerosis in American Indians: the strong heart family study. Atherosclerosis. (2014) 233:165-71. doi: 10.1016/j.atherosclerosis.2013.12.007

28. Stephensen CB, Armstrong P, Newman JW, Pedersen TL, Legault J, Schuster GU, et al. ALOX5 gene variants affect eicosanoid production and response to fish oil supplementation. J Lipid Res. (2011) 52:9911003. doi: 10.1194/jlr.P012864

29. Blum CB. Dynamics of apolipoprotein E metabolism in humans. J Lipid Res. (1982) 23:1308-16. doi: 10.1016/S0022-2275(20)38036-6

30. Abondio P, Sazzini M, Garagnani P, Boattini A, Monti D, Franceschi C, et al. The genetic variability of APOE in different human populations and its implications for longevity. Genes. (2019) 10:222. doi: 10.3390/genes10030222

31. Rebeck GW. The role of APOE on lipid homeostasis and inflammation in normal brains. J Lipid Res. (2017) 58:1493-9. doi: 10.1194/jlr.R075408

32. Minihane AM, Khan S, Leigh-Firbank EC, Talmud P, Wright JW, Murphy $\mathrm{MC}$, et al. ApoE polymorphism and fish oil supplementation in subjects with an atherogenic lipoprotein phenotype. Arterioscler Thromb Vasc Biol. (2000) 20:1990-7. doi: 10.1161/01.ATV.20.8.1990

33. Grimm MOW, Michaelson DM, Hartmann T. Omega-3 fatty acids, lipids, and apoE lipidation in Alzheimer's disease: a rationale for multi-nutrient dementia prevention. J Lipid Res. (2017) 58:2083-101. doi: 10.1194/jlr.R076331

34. Fallaize R, Celis-Morales C, Macready AL, Marsaux CF, Forster H, O'Donovan C, et al. The effect of the apolipoprotein $\mathrm{E}$ genotype on response to personalized dietary advice intervention: findings from the Food4Me randomized controlled trial. Am J Clin Nutr. (2016) 104:82736. doi: 10.3945/ajcn.116.135012

35. Griffin BA, Walker CG, Jebb SA, Moore C, Frost GS, Goff L, et al. APOE4 Genotype exerts greater benefit in lowering plasma cholesterol and apolipoprotein B than wild type (E3/E3), after replacement of dietary saturated fats with low glycaemic index carbohydrates. Nutrients. (2018) 10:1524. doi: 10.3390/nu10101524
36. Chao S, Roberts JS, Marteau TM, Silliman R, Cupples LA, Green RC. Health behavior changes after genetic risk assessment for Alzheimer disease: the REVEAL study. Alzheimer Dis Assoc Disord. (2008) 22:947. doi: 10.1097/WAD.0b013e31815a9dcc

37. Hanson AJ, Bayer JL, Baker LD, Cholerton B, VanFossen B, Trittschuh E, et al. Differential effects of meal challenges on cognition, metabolism, and biomarkers for apolipoprotein E $\varepsilon 4$ carriers and adults with mild cognitive impairment. J Alzheimers Dis. (2015) 48:205-18. doi: 10.3233/JAD-150273

38. Browning LM, Walker CG, Mander AP, West AL, Madden J, Gambell JM, et al. Incorporation of eicosapentaenoic and docosahexaenoic acids into lipid pools when given as supplements providing doses equivalent to typical intakes of oily fish. Am J Clin Nutr. (2012) 96:748-58. doi: 10.3945/ajcn.112.041343

39. Ostermann AI, Willenberg I, Schebb NH. Comparison of sample preparation methods for the quantitative analysis of eicosanoids and other oxylipins in plasma by means of LC-MS/MS. Anal Bioanal Chem. (2015) 407:140314. doi: 10.1007/s00216-014-8377-4

40. Yamada H, Kikuchi S, Hakozaki M, Motodate K, Nagahora N, Hirose M. 8Hydroxyeicosapentaenoic acid decreases plasma and hepatic triglycerides via activation of peroxisome proliferator-activated receptor alpha in high-fat dietinduced obese mice. J Lipids. (2016) 2016:7498508. doi: 10.1155/2016/7498508

41. Yamada H, Oshiro E, Kikuchi S, Hakozaki M, Takahashi H, Kimura K. Hydroxyeicosapentaenoic acids from the Pacific krill show high ligand activities for PPARs[S]. J Lipid Res. (2014) 55:895-904. doi: 10.1194/jlr.M047514

42. Kloske CM, Wilcock DM. The important interface between apolipoprotein $\mathrm{E}$ and neuroinflammation in Alzheimer's disease. Front Immunol. (2020) 11:754. doi: 10.3389/fimmu.2020.00754

43. Huebbe P, Lodge JK, Rimbach G. Implications of apolipoprotein E genotype on inflammation and vitamin E status. Mol Nutr Food Res. (2010) 54:62330. doi: 10.1002/mnfr.200900398

44. Liang S, Steffen LM, Steffen BT, Guan W, Weir NL, Rich SS, et al. APOE genotype modifies the association between plasma omega-3 fatty acids and plasma lipids in the multi-ethnic study of atherosclerosis (MESA). Atherosclerosis. (2013) 228:181-7. doi: 10.1016/j.atherosclerosis.2013.02.004

45. Tomaszewski N, He X, Solomon V, Lee M, Mack WJ, Quinn JF, et al. Effect of APOE genotype on plasma docosahexaenoic acid (DHA), eicosapentaenoic acid, arachidonic acid, and hippocampal volume in the alzheimer's disease cooperative study-sponsored DHA clinical trial. J Alzheimers Dis. (2020) 74:975-90. doi: 10.3233/JAD-191017

46. Plourde M, Vohl MC, Vandal M, Couture P, Lemieux S, Cunnane SC. Plasma n-3 fatty acid response to an n-3 fatty acid supplement is modulated by apoE epsilon4 but not by the common PPAR-alpha L162V polymorphism in men. Br J Nutr. (2009) 102:1121-4. doi: 10.1017/S000711450938215X

47. Chouinard-Watkins R, Conway V, Minihane AM, Jackson KG, Lovegrove JA, Plourde M. Interaction between BMI and APOE genotype is associated with changes in the plasma long-chain-PUFA response to a fish-oil supplement in healthy participants. Am J Clin Nutr. (2015) 102:50513. doi: 10.3945/ajcn.114.103507

48. Caslake MJ, Miles EA, Kofler BM, Lietz G, Curtis P, Armah CK, et al. Effect of sex and genotype on cardiovascular biomarker response to fish oils: the FINGEN Study. Am J Clin Nutr. (2008) 88:618-29. doi: 10.1093/ajcn/88.3.618

49. Yassine HN, Rawat V, Mack WJ, Quinn JF, Yurko-Mauro K, BaileyHall E, et al. The effect of APOE genotype on the delivery of DHA to cerebrospinal fluid in Alzheimer's disease. Alzheimers Res Ther. (2016) 8:25. doi: 10.1186/s13195-016-0194-x

50. Theken KN, Deng Y, Schuck RN, Oni-Orisan A, Miller TM, Kannon MA, et al. Enalapril reverses high-fat diet-induced alterations in cytochrome P450mediated eicosanoid metabolism. Am J Physiol Endocrinol Metab. (2012) 302:E500-9. doi: 10.1152/ajpendo.00370.2011

51. Allen-Redpath K, Aldrovandi M, Lauder SN, Gketsopoulou A, Tyrrell VJ, Slatter DA, et al. Phospholipid membranes drive abdominal aortic aneurysm development through stimulating coagulation factor activity. Proc Natl Acad Sci USA. (2019) 116:8038-47. doi: 10.1073/pnas.1814409116

52. Martinsen A, Tejera N, Vauzour D, Harden G, Dick J, Shinde S, et al. Altered SPMs and age-associated decrease in brain DHA in APOE4 female mice. FASEB J. (2019) 33:10315-26. doi: 10.1096/fj.201900423R

53. Shearer GC, Borkowski K, Puumala SL, Harris WS, Pedersen TL, Newman JW. Abnormal lipoprotein oxylipins in metabolic syndrome and partial 
correction by omega-3 fatty acids. Prostaglandins Leukot Essent Fatty Acids. (2018) 128:1-10. doi: 10.1016/j.plefa.2017.10.006

54. Shearer GC, Walker RE. An overview of the biologic effects of omega- 6 oxylipins in humans. Prostaglandins Leukot Essent Fatty Acids. (2018) 137:2638. doi: 10.1016/j.plefa.2018.06.005

55. Altenburg M, Arbones-Mainar J, Johnson L, Wilder J, Maeda N. Human LDL receptor enhances sequestration of ApoE4 and VLDL remnants on the surface of hepatocytes but not their internalization in mice. Arterioscler Thromb Vasc Biol. (2008) 28:1104-10. doi: 10.1161/ATVBAHA.108.164863

56. Phillips MC. Apolipoprotein E isoforms and lipoprotein metabolism. IUBMB Life. (2014) 66:616-23. doi: 10.1002/iub.1314

57. Skulas-Ray AC, Wilson PWF, Harris WS, Brinton EA, KrisEtherton PM, Richter CK, et al. Omega-3 fatty acids for the management of hypertriglyceridemia: a science advisory from the American Heart Association. Circulation. (2019) 140:e67391. doi: 10.1161/CIR.0000000000000709

58. Demler OV, Liu Y, Luttmann-Gibson H, Watrous JD, Lagerborg KA, Dashti $\mathrm{H}$, et al. One-year effects of Omega-3 treatment on fatty acids, oxylipins, and related bioactive lipids and their associations with clinical lipid and inflammatory biomarkers: findings from a substudy of the Vitamin D and Omega-3 Trial (VITAL). Metabolites. (2020) 10:431. doi: 10.3390/metabo10110431

59. Fallaize R, Carvalho-Wells AL, Tierney AC, Marin C, Kieć-Wilk B, Dembińska-Kieć A, et al. APOE genotype influences insulin resistance, apolipoprotein $\mathrm{CII}$ and CIII according to plasma fatty acid profile in the Metabolic Syndrome. Sci Rep. (2017) 7:6274. doi: 10.1038/s41598-017-05802-2

60. Wu L, Zhang X, Zhao L. Human ApoE isoforms differentially modulate brain glucose and ketone body metabolism: implications for alzheimer's disease risk reduction and early intervention. J Neurosci. (2018) 38:666581. doi: 10.1523/JNEUROSCI.2262-17.2018

61. Williams HC, Farmer BC, Piron MA, Walsh AE, Bruntz RC, Gentry MS, et al. APOE alters glucose flux through central carbon pathways in astrocytes. Neurobiol Dis. (2020) 136:104742. doi: 10.1016/j.nbd.2020.104742

62. Chouinard-Watkins R, Plourde M. Fatty acid metabolism in carriers of apolipoprotein E epsilon 4 allele: is it contributing to higher risk of cognitive decline and coronary heart disease? Nutrients. (2014) 6:445271. doi: 10.3390/nu6104452

63. Risner ME, Saunders AM, Altman JF, Ormandy GC, Craft S, Foley IM, et al. Efficacy of rosiglitazone in a genetically defined population with mild-to-moderate Alzheimer's disease. Pharmacogenomics J. (2006) 6:24654. doi: 10.1038/sj.tpj.6500369

64. Arbones-Mainar JM, Johnson LA, Altenburg MK, Kim H-S, Maeda N. Impaired adipogenic response to thiazolidinediones in mice expressing human apolipoproteinE4. FASEB J. (2010) 24:3809-18. doi: 10.1096/fj.10-159517

65. Jofre-Monseny L, Minihane AM, Rimbach G. Impact of apoE genotype on oxidative stress, inflammation and disease risk. Mol Nutr Food Res. (2008) 52:131-45. doi: 10.1002/mnfr.200700322

66. Tao Q, Ang TFA, DeCarli C, Auerbach SH, Devine S, Stein TD, et al. Association of chronic low-grade inflammation with risk of alzheimer disease in ApoE4 carriers. JAMA Netw Open. (2018) 1:e183597. doi: 10.1001/jamanetworkopen.2018.3597

67. Kofler BM, Miles EA, Curtis P, Armah CK, Tricon S, Grew J, et al. Apolipoprotein E genotype and the cardiovascular disease risk phenotype: impact of sex and adiposity (the FINGEN study). Atherosclerosis. (2012) 221:467-70. doi: 10.1016/j.atherosclerosis.2012.01.042

68. Huo Y, Zhao L, Hyman MC, Shashkin P, Harry BL, Burcin T, et al. Critical role of macrophage 12/15-lipoxygenase for atherosclerosis in apolipoprotein E\&\#x2013; deficient mice. Circulation. (2004) 110:202431. doi: 10.1161/01.CIR.0000143628.37680.F6

69. Qiu H, Gabrielsen A, Agardh HE, Wan M, Wetterholm A, Wong CH, et al. Expression of 5-lipoxygenase and leukotriene A4 hydrolase in human atherosclerotic lesions correlates with symptoms of plaque instability. Proc Natl Acad Sci USA. (2006) 103:8161-6. doi: 10.1073/pnas.0602414103

70. Chinnici CM, Yao Y, Ding T, Funk CD, Praticò D. Absence of 12/15 lipoxygenase reduces brain oxidative stress in apolipoprotein E-deficient mice. Am J Pathol. (2005) 167:1371-7. doi: 10.1016/S0002-9440(10)61224-2

71. Carracedo M, Artiach G, Arnardottir H, Bäck M. The resolution of inflammation through omega-3 fatty acids in atherosclerosis, intimal hyperplasia, and vascular calcification. Semin Immunopathol. (2019) 41:75766. doi: 10.1007/s00281-019-00767-y

72. Devassy JG, Leng S, Gabbs M, Monirujjaman M, Aukema HM. Omega-3 Polyunsaturated fatty acids and oxylipins in neuroinflammation and management of alzheimer disease. Adv Nutr. (2016) 7:905-16. doi: 10.3945/an.116.012187

73. Keenan AH, Pedersen TL, Fillaus K, Larson MK, Shearer GC, Newman JW. Basal omega-3 fatty acid status affects fatty acid and oxylipin responses to high-dose n3-HUFA in healthy volunteers. J Lipid Res. (2012) 53:1662-9. doi: 10.1194/jlr.P0 25577

74. Spector AA, Kim HY. Cytochrome P450 epoxygenase pathway of polyunsaturated fatty acid metabolism. Biochim Biophys Acta. (2015) 1851:356-65. doi: 10.1016/j.bbalip.2014. 07.020

75. Calder PC. Eicosapentaenoic and docosahexaenoic acid derived specialised pro-resolving mediators: concentrations in humans and the effects of age, sex, disease and increased omega-3 fatty acid intake. Biochimie. (2020) 178:10523. doi: 10.1016/j.biochi.2020.08.015

76. Yassine HN, Finch CE. APOE Alleles and diet in brain aging and alzheimer's disease. Front Aging Neurosci. (2020) 12:150. doi: 10.3389/fnagi.2020.00150

Conflict of Interest: The authors declare that the research was conducted in the absence of any commercial or financial relationships that could be construed as a potential conflict of interest.

Publisher's Note: All claims expressed in this article are solely those of the authors and do not necessarily represent those of their affiliated organizations, or those of the publisher, the editors and the reviewers. Any product that may be evaluated in this article, or claim that may be made by its manufacturer, is not guaranteed or endorsed by the publisher.

Copyright (C) 2021 Saleh, West, Ostermann, Schebb, Calder and Minihane. This is an open-access article distributed under the terms of the Creative Commons Attribution License (CC BY). The use, distribution or reproduction in other forums is permitted, provided the original author(s) and the copyright owner(s) are credited and that the original publication in this journal is cited, in accordance with accepted academic practice. No use, distribution or reproduction is permitted which does not comply with these terms. 